\title{
Ethnoveterinary plants of Ankober District, North Shewa Zone, Amhara Region, Ethiopia
}

Ermias Lulekal ${ }^{1,2^{*}}$, Zemede Asfaw ${ }^{2}$, Ensermu Kelbessa ${ }^{2}$ and Patrick Van Damme ${ }^{1,3}$

\begin{abstract}
Background: Traditional herbal preparations for addressing veterinary problems have been applied in Ankober District, Ethiopia, for generations. However, the millennia-old ethnoveterinary knowledge of the community, and the plants are subjected to loss without being scientifically documented due to anthropogenic and environmental threats. Hence, this study aims at providing a comprehensive documentation on ethnoveterinary plant knowledge of the people in order to preserve the fast-eroding knowledge and resources of the area.

Methods: Semi-structured interviews, focus group discussions, participant observation and walk-in-the-woods methods were used to gather ethnoveterinary data. Informant Consensus Factor (ICF) and Fidelity level (FL) values were calculated using quantitative approaches so as to check the level of informants' agreement on plant use and healing potential of ethnoveterinary medicinal plant species, respectively. Indigenous knowledge on use of medicinal plants for ethnoveterinary purposes among different informant groups was compared using One-way ANOVA and t-tests.

Results: A total of 51 plant species representing 50 genera and 35 botanical families used in the treatment of 33 different ailments were identified. Medicinal plant species belonging to families Asteraceae, Asclepiadaceae, Euphorbiaceae and Ranunculaceae were reported to be of frequent use in the local ethnoveterinary medical system. Roots (65\%, 33 species) were most often utilized for remedy preparation. Highest ICF values were recorded for gastro-intestinal (0.71) ailments depicting best agreement on knowledge of medicinal plants used to treat aliments in this category. Embelia schimperi Vatke showed highest fidelity level value (90\%) to treat gastro-intestinal diseases showing conformity of knowledge on this species' healing potential. Significant difference $(P<0.05)$ was observed in average number of therapeutic plants reported by senior members of the community than younger groups. Embelia schimperi Vatke and Rubus steudnerii Schweinf. were the most-preferred species to treat diarrhoea.
\end{abstract}

Conclusion: The study indicated that indigenous knowledge on ethnoveterinary medicinal plant use is still rich and active in the District. Species with recorded highest consensus for curative role are a useful pool for further phytochemical and pharmacological validation for better utilization. Declining wild medicinal flora of the area calls for implementation of a coordinated complementary in situ and ex situ conservation strategy.

Keywords: Ethnomedicine, Fidelity level, Traditional knowledge, Informants consensus factor, Preference ranking, Traditional healers

\footnotetext{
* Correspondence: ermiasmulekal.molla@Ugent.be

${ }^{1}$ Laboratory for Tropical and Subtropical Agriculture and Ethnobotany,

Department of Plant Production, Faculty of Bio-Science Engineering, Ghent

University, Coupure links 653, 9000 Ghent, Belgium

2Department of Plant Biology and Biodiversity Management, College of

Natural sciences, Addis Ababa University, P.O. Box 3434, Addis Ababa,

Ethiopia

Full list of author information is available at the end of the article
}

\section{Biomed Central}

(c) 2014 Lulekal et al.; licensee BioMed Central Ltd. This is an Open Access article distributed under the terms of the Creative Commons Attribution License (http://creativecommons.org/licenses/by/2.0), which permits unrestricted use, distribution, and reproduction in any medium, provided the original work is properly credited. The Creative Commons Public Domain Dedication waiver (http://creativecommons.org/publicdomain/zero/1.0/) applies to the data made available in this article, unless otherwise stated. 


\section{Background}

Livestock husbandry is a mainstay in the livelihood of more than $70 \%$ of Ethiopians [1]. Since the country's economy is mainly based on agriculture, the livestock subsector is positively important in that it contributes about $16 \%$ of the national GDP and $45 \%$ of agricultural GDP [2]. About $90 \%$ of the crop production in Ethiopia depends on animal draft power [3]. Livestock also offers the only way of survival in many harsh environments on top of serving as a driving force of food security in the country [4]. Ethiopia has the highest number of livestock in Africa which is estimated to 53.3 million heads of cattle, 25.5 million sheep, 22.7 million goats, 5.7 million donkeys, 2 million horses, 1.1 million camels and 49.2 million chicken; hence the country is listed among the top 10 countries in the continent known for their livestock wealth [5]. Despite this large number of livestock and its important economic potential, the sector has not developed beyond a subsistence type of venture, whereas it also remained with low outputs for different reasons of which animal diseases are among the top factors [6].

Diseases are the prime causes of poor livestock productivity in many developing countries [7]. According to Admassu [8], the aggregate annual economic loss in Ethiopia from livestock diseases (through direct mortality, and reduced productive and reproductive performance) was estimated at US\$ 150 million. Fromsa and Jobre [9] estimated annual economic loss from bovine hydatidosis to be at US\$ 101 million in the country. Although disease-free livestock products (for in-house consumption and export purposes) are mandatory to ensure consumer's health and to reap more earnings from the sector, it is hardly possible to provide an overall conventional veterinary medical services in Ethiopia and most developing countries, hence the sector remained with low output [4]. According to Duguma [6], inadequate veterinary health professionals, scarce and erratic supply of veterinary drugs, high cost of equipment and drugs, absence of government-based livestock health policies, presence of counter-productive livestock health policies and poor infrastructure are mentioned as some of the major factors that made livestock raisers in developing countries to rely more on ethnoveterinary medicine than the modern medical system.

Settled and nomadic livestock raisers from many countries have developed their own indigenous knowledge on ethnoveterinary practices through age-old cultural contact with curative plants, trial and error experiments and empirical observations to treat various livestock ailments [6]. Traditional knowledge on use of medicinal plants, however, is subjected to loss in the absence of incessant cultural interaction with medicinal species [10,11]. Reliance on ethnoveterinary knowledge in different countries is mostly reinforced by the strong dependence on livestock for livelihood and the richness in cultural history, ancestral knowledge and biodiversity [12]. According to Cetinkaya [13], demographic, economic, socio-political, ecological, religious and cultural factors existing in a community are key drivers shaping traditional knowledge in a given society.

Millennia-old traditional knowledge on ethnoveterinary folk medicine has been recognised during the 32nd session of UNESCO as one of the important components of indigenous cultural heritage that need to be studied, sustained and protected [14]. Since then, scientific studies and documentation of indigenous knowledge on ethnoveterinary medicinal plants have been initiated in many countries; to mention but few: Kenya [15], Mediterranean areas of Albania, Italy, Morocco, Spain, Egypt, Greece, Algeria and Cyprus [16], Canada [17], South Africa [18], Pakistan [19], Uganda [12], Brazil [20], Argentina [21], India [22], Nigeria [23] and Spain [24] and Germany [25]. However, the effort is still quite insignificant when compared to the undocumented global ethnoveterinary plant lore.

In Ethiopia, ethnoveterinary practices were reported to be the only options to cure livestock ailments till the advent of modern veterinary services which were started as late as 1908 [8]. Although a gain from the richest livestock wealth of the country is directly related to safeguarding livestock health, conventional veterinary medical system is yet very poor in the country [26]. Abebe [27], stated that traditional plant remedies are major sources of therapeutics for nearly 90\% of the livestock population in Ethiopia. Moreover, about $95 \%$ of all forms of traditional medicinal preparations in the country are also reported to be of plant origin [28]. Thus, the deep-seated custom of using medicinal plants led Ethiopian cultural communities to know medicinal properties of many plants that are used to treat livestock ailments [29]. However, the rich indigenous knowledge on many of the traditional plant remedies is subjected to loss as it has mainly been passed orally for generations without being properly nor scientifically documented [30].

Despite the significant role played by ethnoveterinary plants for treating livestock ailments in both settled and pastoralist areas of Ethiopia, a very limited attempt has been done to explore, document and promote these widely used ethnoveterinary plants in the country. Moreover, Ethiopian medicinal plants and the associated indigenous knowledge are continuously threatened due to factors like deforestation, overexploitation, overgrazing, habitat loss and degradation, agricultural land expansion and acculturation [31]. Hence, it is a timely endeavour to document, promote and conserve the country's ethnoveterinary medicinal plant lore. Such documents are important to define and maintain cultural identity of the people [13] in addition to serving as keys towards establishing people-centred natural resource management 
systems [32], and potentials for scientific discovery of new lead compounds used in the development of modern drugs [33].

Although Ethiopian ethnoveterinary medicinal inventories including those of [7,34-44], have attempted to document veterinary importance of traditional medicinal plants in some cultural groups, it is found insignificant when compared to the 85 different ethnolinguistic communities found in the country, which have remained largely unexplored. Hence, the present research aims to fill this gap by documenting the wealth of indigenous knowledge on utilization, management and conservation of ethnoveterinary plants used in Ankober District, north Shewa Zone, Ethiopia, which has never been explored for its ethnoveterinary wealth. It also aims to identify and document marketable medicinal plants of the District so as to identify the economic potential of medicinal plants in the area. In addition, it aims to select candidate ethnoveterinary plant species with high informants' consensus and fidelity level values for further phytochemical and pharmacological analyses in subsequent studies.

\section{Methods}

\section{Study area and ethnographic background}

This study was conducted in Ankober Distrcit, located at $9^{\circ} 22^{\prime} 0^{\prime \prime}-9^{\circ} 45^{\prime} 0^{\prime \prime} \mathrm{N}$ and $039^{\circ} 40^{\prime} 0^{\prime \prime}-039^{\circ} 53^{\prime} 0^{\prime \prime} \mathrm{E}$ in north Shewa Zone of Amhara National Regional State, north-central Ethiopia (Figure 1). The District is perched on the eastern escarpment of the Ethiopian highlands and located at $172 \mathrm{~km}$ north of Addis Ababa, the Ethiopian capital, and $42 \mathrm{~km}$ to the east of Debre Berhan town (the north Shewa Zone capital). Ankober District is bordered in the north by Tarmaber District, south by Asagirt District and west by Basonaworana District of Amhara Region. The eastern part shares its border with Gachine Special District of the Afar Region [45]. Elevation in Ankober District ranges from $1300 \mathrm{~m}$ asl near Addis Alem area to $3700 \mathrm{~m}$ asl at Kundi Mountain. Annual rainfall in the District ranges 1000 to $1400 \mathrm{~mm}$ and cold temperature is

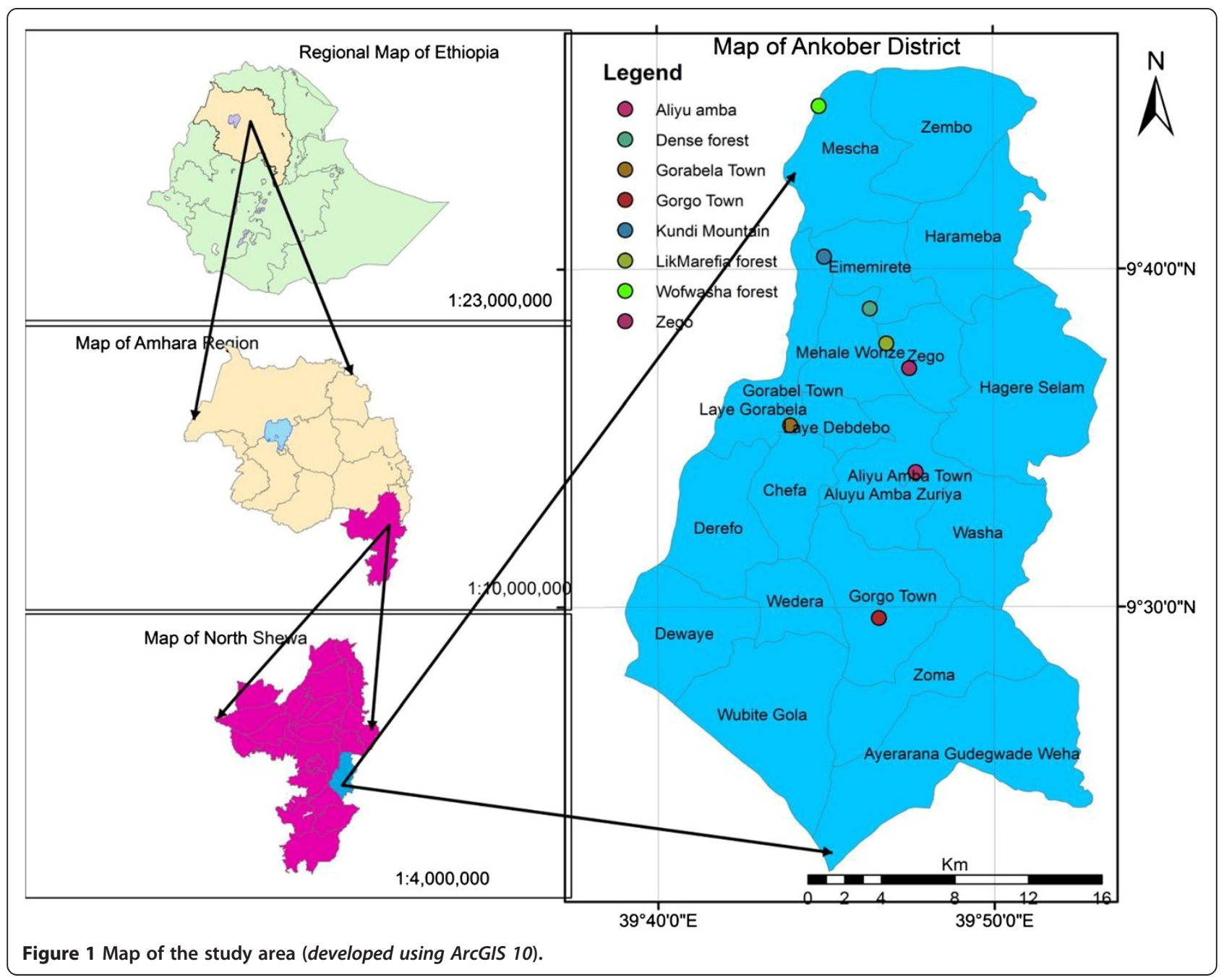


prominent for most of the year [46]. The main administrative centre of the District is located at Gorabela/Ankober town that has historical significance as it has been the seat of the Ethiopian emperors from 1270 for centuries [45].

The indigenous people inhabiting the area belong to the Amhara ethnic group. They speak Amharic language, the national language of Ethiopia. The District has a total population of $83,260(42,180$ men and 41,080 women) of whom only 6,272 (7.5\%) are urban inhabitants [5]. Ankober has a population density of 113.72 individuals $/ \mathrm{km}^{2}$, which is slightly less than the north Shewa Zone average of 115.3 persons $/ \mathrm{km}^{2}$ [46]. About 92.52\% of the people in Ankober belong to the Ethiopian Orthodox Tewahdo Christianity and $7.41 \%$ are Muslims.

Ankober District homes Dense and Likmarefia forests and part of the Wof Washa natural forest. These forests are rich in biodiversity and harbour economically important tree species including Hagenia abyssinica (Bruce) J.F. Gmel., Olea europaea L. subsp. cuspidata (Wall. ex G. Don), Juniperus procera L., Podocarpus falcatus (Thunb.) Mirb. and Nuxia congesta R. Br. ex Fresen [47]. They are also home to very diverse wildlife and bird species including the IUCN red-listed endemic bird species Serinus ankoberensis [48].

\section{Livestock population and status of veterinary services in the District}

The livestock population in the District was estimated to 61,499 heads of cattle, 33,265 sheep, 54,493 goats, 8,802 donkeys, 395 horses, 244 camels, 221 mules and 47,756 chicken in the year 2012 [46]. At the same time, there were only seven rural veterinary clinics in the District located at Mescha, Haramba, Gorgo, Aliyu Amba, Wedera, Derefo and Lay Gorebela kebeles (Lowest administrative units in Ethiopia). The remaining 15 kebeles did not have veterinary clinics at all. There were only eight veterinarians (1 DVM, 2 Bsc and 2 Diploma holders with 3 assistants) working in the District in 2012 [46]. This was found insufficient to provide proper health services for a total of more than 200,000 heads present in the District [46]. Blackleg, pasteurellosis, Newcastle disease, lumpy skin disease, foot and mouth disease, African horse sickness and various parasitic diseases are reported as the most common disease types affecting the District [46]. The local people in Ankober have long been dependent on traditional medicinal plants accessed from natural forest patches, cultivated land and field margins to manage various animal health problems.

\section{Selection of informants}

Representative informants and knowledgeable traditional medicine practitioners of the District were selected using systematic random and purposive sampling approaches in the manner described by Martin [49]. The total number of informants involved in the ethnomedicinal survey was 352 (235 men and 117 women). Informants' ages ranged 20- 89years (122 were between 20-39 whereas 230 were $\geq 40$ years old). All twenty-two kebeles of Ankober District were included in this investigation. Peer-recommendations from community members, elderly people and knowledgeable inhabitants helped in nominating 88 traditional herbalists (68 men and 20 women) who participated as key respondents following Davis and Wagner [50], whereas general informants were sampled during random visits made to houses in the study kebeles. Informed consent was obtained from each informant who participated in this study after explaining the purpose of the study and assuring them of the most responsible judicial use in the resulting information before the start of interviews.

\section{Ethnobotanical data collection}

Ethnoveterinary data were collected during six different field visits conducted between June 2009 and May 2011. Data were collected in different seasons over the different years with the objective of including all kebeles in the District and collecting plant specimens during the respective flowering seasons. Market survey and checking of reliability of informants' medicinal plant use knowledge were conducted between 29 December 2012 and 9 February 2013.

Semi-structured interviews, focus group discussions, participant observation and walk-in-the-woods were used to collect ethnoveterinary data as described by Martin [49], Cotton [51]. Informants were interviewed individually in the local Amharic language. Semi-structured interviews addressed questions regarding name, age, gender, level of education, occupation, religion and ethnic background of each informant. The individual semi-structured interviews included local names of medicinal plants, ailments treated, habitat of the species, distance from the house to gathering sites, seasonality of species, marketability of species, degree of management (wild/cultivated), abundance, parts used, condition of plant part used (fresh/dried), other ingredients or additives (if any), methods of remedy preparation, remedy preservation (storage), dosage prescriptions, routes of remedy administration, noticeable adverse effects of remedies, use of antidotes for adverse effects, taboos/beliefs related to collection and use of plants, source of knowledge, method of indigenous knowledge transfer, other uses of species mentioned, existing threats and traditional conservation practices (if any), and in the case of traditional healers number of years of service as healer and income earned per animal treated for an ailment following the methods used by previous researchers [49,51-53].

All semi-structured interviews were followed by independent walk-in-the-woods activities, which gave an 
opportunity for more discussion with individual informants and the practical identification and collection of traditionally used medicinal plants in their natural environment. This method was combined with participant observation through which reliability of the information collected was ascertained on collection methods and preparation of specific remedial parts as described by Cotton [51], Alexiades [52]. In addition, focus group (one focus group per kebele with average number of 5 participants) discussions were undertaken to gain further information on medicinal plants knowledge of the community and prove the reliability of the data collected through semi-structured interviews [49].

Preference ranking was conducted using fifteen key informants (ten men and five women) in the manner recommended by Martin [49] to identify the-most preferred species used for treating the most commonly reported gastro-intestinal disease in the area. Major markets of Ankober District i.e., Gorebella, Aliyuamba, Gorgo, Haramba, Derefo, and Zego were surveyed for availability, price and unit of measurement of marketable medicinal plants (if any).

Voucher specimens of medicinal plants were collected on the field with the help of traditional healers and local field assistants. Specimens were dried, numbered, labelled, pressed, identified and deposited at the National Herbarium (ETH) in Addis Ababa University. Identification of specimens was performed both in the field and later at ETH using taxonomic keys and floras [54-61] and by comparison with authenticated herbarium specimens.

\section{Data analysis}

Ethnoveterinary data on medicinal plants used in Ankober District were entered in an excel spreadsheet (Microsoft corporation, 2007) and organised for statistical analysis. Descriptive statistics was applied to compute the number and percentage of species, genera and families of ethnoveterinary medicinal plants, their growth forms, proportions of parts harvested, modes of remedy preparation and routes of administration. Preferences of ethnoveterinary plant species used to treat the commonly reported livestock ailments in the study area were ranked by adding the values/scores of preferences given by respective informants so as to identify the most-preferred medicinal plant species to treat the most frequently reported disease type in the area following the relevant standard methods $[49,52]$.

Informant Consensus Factor (ICF) values [62] were calculated to determine the most important livestock ailment categories in the District and identify potentially effective medicinal plant species in respective disease categories. Accordingly, reported traditional remedies and corresponding livestock ailments occurring in the District were categorized into eight disease categories and the ICF values were obtained by computing number of use citations in each disease category $\left(n_{\mathrm{ur}}\right)$ minus the number of times species used $\left(\mathrm{n}_{\mathrm{t}}\right)$, divided by the number of use citations in each category minus one.

$$
\mathrm{ICF}=\mathrm{n}_{\mathrm{ur}-} \mathrm{n}_{\mathrm{t}} / \mathrm{n}_{\mathrm{ur}-1}
$$

An index of Fidelity Level (FL) given by $\mathrm{FL}=\mathrm{Ip} / \mathrm{Iu} \mathrm{X}$ 100 [52], where Ip is the number of informants who independently indicated the use of a species for treating a particular disease, and Iu the total number of informants who reported the plant for any given disease was used to determine the relative healing potential of reported ethnoveterinary plant.

Traditional knowledge dynamics on use of medicinal plants by men and women, young to middle aged (2339 years) and elderly (40-89 years); literate (completed at least primary education) and illiterate; knowledgeable (key) and local (encountered randomly) informants was compared using $t$-test and one way ANOVA at 95\% confidence level between means, since data are normally distributed, using KyPlot 5.0 software.

\section{Results \\ Ethnoveterinary medicinal plant diversity in Ankober District}

A total of 51 ethnoveterinary medicinal plant species representing 50 genera and 35 botanical families were identified in the District (Table 1). Thirty-one percent of the botanical families were represented by more than one species. The highest number of species was recorded for family Asteraceae (4 species, 8\%) followed by Asclepiadaceae, Euphorbiaceae and Ranunculaceae (3, 6\% each). Seven of the reported botanical families i.e., Fabaceae, Lamiaceae, Menispermaceae, Myrsinaceae, Plantaginaceae, Rubiaceae and Solanaceae were represented by 2 (4\%) species each. The remaining 24 (69\%) families had a single species representation. About 6\% (3 species) of the medicinal plant species are endemic to Ethiopia (Table 1).

Regarding the growth forms of plants of ethnoveterinary importance, there were more shrubs (23 species, $45 \%)$ followed by herbs $(13,25 \%)$, trees $(10,20 \%)$ and climbers $(5,10 \%)$. About $80 \%$ (41 species) of medicinal plants were those that are harvested from the wild environment, whereas $6 \%$ (3 species) from cultivation and the remaining $14 \%$ (7 species) were reported to be collected from both wild and cultivated sources. Deforestation (reported by $89 \%$ of informants), agricultural expansion (80\%), charcoal making and firewood collection (33\% collectively) and overgrazing (29\%) were claimed as major factors affecting the medicinal plant wealth of the area. 
Table 1 List of ethnoveterinary medicinal plants used for treatment of livestock ailments: scientific name; family; local name; growth form; ailment treated; plant parts used; condition of plant part uses; methods of preparation and application, route of administration, plant part mixed with and voucher number

\begin{tabular}{|c|c|c|c|c|c|c|c|c|c|c|}
\hline Scientific name & Family & $\begin{array}{l}\text { Local (Amharic) } \\
\text { name }\end{array}$ & Growth form & Ailment treated & Part used & CPU & $\begin{array}{l}\text { MP } \\
\text { AP }\end{array}$ & RA & $\begin{array}{l}\text { Part mixed } \\
\text { with }\end{array}$ & $\begin{array}{l}\text { Voucher no } \\
\text { ermiasLX }\end{array}$ \\
\hline \multirow[t]{3}{*}{ Achyranthes aspera $\mathrm{L}$. } & Amaranthaceae & Telenj & $\mathrm{H}$ & Nasal infection & $R$ & $\mathrm{~F}$ & 5 & $\mathrm{Na}$ & & 104 \\
\hline & & & & Ophthalmic infection & L & $\mathrm{F}$ & 4 & Op & & \\
\hline & & & & Minor bleeding & L & $\mathrm{F}$ & 3 & De & & \\
\hline \multirow[t]{2}{*}{$\begin{array}{l}\text { Aeonium leucoblepharum Webb ex A. } \\
\text { Richard }\end{array}$} & Crassulaceae & Yefeyel Dabo & $\mathrm{H}$ & Sore & L & $\mathrm{F}$ & 2 & De & & 885 \\
\hline & & & & $\begin{array}{l}\text { Retained placenta/fetal } \\
\text { membrane }\end{array}$ & L & $\mathrm{F}$ & 5 & $\mathrm{O}$ & & \\
\hline \multirow[t]{8}{*}{ Allium sativum $\mathrm{L}$. } & Alliaceae & Nech Shinkurt & $\mathrm{H}$ & Blackleg & $\mathrm{BU}$ & F/D & 5 & $\mathrm{O}$ & 8,11 & 828 \\
\hline & & & & Dermatophilosis & $\mathrm{BU}$ & F/D & 2 & De & & \\
\hline & & & & Mange & $\mathrm{BU}$ & $F / D$ & 2 & $\mathrm{De}$ & & \\
\hline & & & & Scabies & BU & F/D & 2 & De & & \\
\hline & & & & Ringworm & $\mathrm{BU}$ & F/D & 2 & De & & \\
\hline & & & & Parasitic leech & $\mathrm{BU}$ & F/D & 5 & $\mathrm{Na}$ & & \\
\hline & & & & Lice infestation in chicken & $\mathrm{BU}$ & $\mathrm{F}$ & 4 & $\mathrm{De}$ & & \\
\hline & & & & Helminthiasis & $L$ & $\mathrm{~F}$ & 5 & $\mathrm{O}$ & & \\
\hline \multirow[t]{2}{*}{ Apodytes dimidiata E. Mey. ex Arn } & Icacinaceae & $\begin{array}{l}\text { Yetemenja Inchet/ } \\
\text { Donga }\end{array}$ & $\mathrm{T}$ & Rabies & SB & $\mathrm{F}$ & 5 & 0 & 5 & 887 \\
\hline & & & & African horse sickness & SB & $\mathrm{F}$ & 5 & $\mathrm{O}$ & & \\
\hline Asparagus africanus Lam. & Asparagaceae & Seritie & $\mathrm{S}$ & Coccidiosis & $\mathrm{R}$ & $\mathrm{F}$ & 1 & $\mathrm{O}$ & 7 & 189 \\
\hline Calotropis procera (Ait.) Aitf & Asclepiadaceae & Kimbo & $\mathrm{H}$ & Mange & $\mathrm{R}$ & $\mathrm{F}$ & 4 & De & & 894 \\
\hline \multirow[t]{5}{*}{ Calpurnia aurea (Ait.) Benth. } & Fabaceae & Digita & $\mathrm{S}$ & Tick infestation & L & $\mathrm{F}$ & 4 & $\mathrm{De}$ & & 76 \\
\hline & & & & Helminthiasis & L & $\mathrm{F}$ & 5 & $\mathrm{O}$ & & \\
\hline & & & & Snake bite & $R$ & $\mathrm{~F}$ & 5 & $\mathrm{O}$ & 3,11 & \\
\hline & & & & Sore & $\mathrm{L}$ & F/D & 3 & De & & \\
\hline & & & & Parasitic leech & $\mathrm{BU}$ & F/D & 5 & $\mathrm{Na}$ & & \\
\hline \multirow[t]{2}{*}{ Carissa spinarum L. } & Apocynaceae & Agam & $\mathrm{S}$ & Helminthiasis & $\mathrm{R}$ & $\mathrm{F}$ & 4 & $\mathrm{O}$ & & 16 \\
\hline & & & & Parasitic leech & L & $\mathrm{F}$ & 4 & De & & \\
\hline \multirow[t]{2}{*}{ Cissampelos mucronata A. Rich. } & Menispermaceae & Ingochit hareg & C & Cowdriosis & $P$ & $\mathrm{~F}$ & 5 & $\mathrm{O}$ & 14 & 888 \\
\hline & & & & CBPP & $P$ & $\mathrm{~F}$ & 5 & O & 40 & \\
\hline \multirow[t]{2}{*}{ Clematis hirsuta Perr. \& Guill. } & Ranunculaceae & Azo hareg & C & Blackleg & $\mathrm{R}$ & $\mathrm{F}$ & 5 & $\mathrm{O}$ & 3,8 & 18 \\
\hline & & & & Pleuropneumoniasis & $\mathrm{L}$ & $\mathrm{F}$ & 5 & O & & \\
\hline
\end{tabular}


Table 1 List of ethnoveterinary medicinal plants used for treatment of livestock ailments: scientific name; family; local name; growth form; ailment treated; plant parts used; condition of plant part uses; methods of preparation and application, route of administration, plant part mixed with and voucher number (Continued)

\begin{tabular}{|c|c|c|c|c|c|c|c|c|c|}
\hline \multirow[t]{5}{*}{ Clutia abyssinica Jaub. \& Spach } & Euphorbiaceae & Feyele Fej & $S$ & Bloody diarrhoea & L & $\mathrm{F}$ & 5 & $\mathrm{O}$ & 10 \\
\hline & & & & Nasal infection & R & $\mathrm{F}$ & 5 & O & \\
\hline & & & & Ophthalmic infection & L & $\mathrm{F}$ & 4 & O & \\
\hline & & & & Mucal diarrhoea & R & $\mathrm{F}$ & 5 & O & \\
\hline & & & & Bloat & L & $\mathrm{F}$ & 5 & O & \\
\hline \multirow[t]{7}{*}{ Croton macrostachyus Del. } & Euphorbiaceae & Bisana & $\mathrm{T}$ & Ringworm & L & $\mathrm{F}$ & 4 & O & 17 \\
\hline & & & & Dermatophilosis & L & $\mathrm{F}$ & 4 & O & \\
\hline & & & & Mange & L & $\mathrm{F}$ & 4 & O & \\
\hline & & & & Scabies & L & $\mathrm{F}$ & 4 & O & \\
\hline & & & & Wound & L & $\mathrm{F}$ & 4 & O & \\
\hline & & & & Minor bleeding & L & $\mathrm{F}$ & 3 & O & \\
\hline & & & & Sore & L & F/D & 3 & O & \\
\hline \multirow[t]{4}{*}{ Cucumis ficifolius A. Rich. } & Cucurbitaceae & Yemdr Imbuay & $\mathrm{H}$ & Coccidiosis & $\mathrm{Fr}, \mathrm{R}$ & $F / D$ & 1 & O & 68 \\
\hline & & & & Cowdriosis & R & $\mathrm{F}$ & 5 & 03 & \\
\hline & & & & Hepatitis & $\mathrm{Fr}$ & F/D & 5 & O & \\
\hline & & & & Wound & $L \& R$ & $\mathrm{~F}$ & 3 & O & \\
\hline \multirow[t]{3}{*}{$\begin{array}{l}\text { Debregeasia saeneb (Forssk.) Hepper \& } \\
\text { Wood }\end{array}$} & Urticaceae & Wenz admek & $S$ & Mange & $\mathrm{R}$ & $\mathrm{F}$ & 2 & O & 890 \\
\hline & & & & Lumpy skin disease & R & $\mathrm{F}$ & 2 & O & \\
\hline & & & & Scabies & R & $\mathrm{F}$ & 2 & O & \\
\hline \multirow[t]{3}{*}{ Discopodium penninervium Hochst. } & Solanaceae & Ameraro & S & Blackleg & L & $\mathrm{F}$ & 5 & O & 606 \\
\hline & & & & Wound & L & $\mathrm{F}$ & 2 & O & \\
\hline & & & & Minor bleeding & L & $\mathrm{F}$ & 3 & O & \\
\hline \multirow[t]{4}{*}{ Dodonaea angustifolia L. f. } & Sapindaceae & Kitkita & S & Bloat & $\mathrm{RB}$ & $F / D$ & 5 & O & 20 \\
\hline & & & & Sudden diarrhoea & R & $\mathrm{F}$ & 5 & O & \\
\hline & & & & Ringworm & $\mathrm{R}$ & $\mathrm{F}$ & 2 & O & \\
\hline & & & & Scabies & $\mathrm{R}$ & $\mathrm{F}$ & 2 & $\mathrm{O}$ & \\
\hline \multirow[t]{3}{*}{ Dracaena afromontana Mildbr. } & Dracenaceae & Merko & S & Blackleg & $\mathrm{R}$ & $\mathrm{F}$ & 5 & O & 892 \\
\hline & & & & Scabies & R & $\mathrm{F}$ & 2 & O & \\
\hline & & & & Dermatophilosis & R & $\mathrm{F}$ & 4 & O & \\
\hline
\end{tabular}
Debregeasia saeneb (Forssk.) Hepper \&
Wood

Discopodium penninervium Hochst.

Dodonaea angustifolia L. f. 
Table 1 List of ethnoveterinary medicinal plants used for treatment of livestock ailments: scientific name; family; local name; growth form; ailment treated; plant parts used; condition of plant part uses; methods of preparation and application, route of administration, plant part mixed with and voucher number (Continued)

\begin{tabular}{|c|c|c|c|c|c|c|c|c|c|c|}
\hline \multirow[t]{2}{*}{ Dregea schimperi (Decne.) Bullock } & \multirow[t]{2}{*}{ Asclepiadaceae } & \multirow[t]{2}{*}{ Yedikula Kend } & \multirow[t]{2}{*}{ C } & Snake bite & $\mathrm{R}$ & $\mathrm{F}$ & 5 & $\mathrm{O}$ & & \multirow[t]{2}{*}{798} \\
\hline & & & & Wound & L & $\mathrm{F}$ & 4 & O & & \\
\hline \multirow[t]{3}{*}{ Embelia schimperi Vatke } & \multirow[t]{3}{*}{ Myrsinaceae } & \multirow[t]{3}{*}{ Inkoko } & \multirow[t]{3}{*}{ C } & Bloat & $\mathrm{Fr}$ & $F / D$ & 5 & $\mathrm{O}$ & & \multirow[t]{3}{*}{505} \\
\hline & & & & Mucal diarrhoea & $\mathrm{Fr}$ & $\mathrm{F}$ & 5 & O & & \\
\hline & & & & Bloody diarrhoea & $\mathrm{Fr}$ & F/D & 5 & O & 4 & \\
\hline \multirow[t]{3}{*}{ Gomphocarpus fruticosus (L.) Ait. F. } & \multirow[t]{3}{*}{ Asclepiadaceae } & \multirow[t]{3}{*}{ Ashkla Hareg } & \multirow[t]{3}{*}{ H } & Bloat & $P$ & $\mathrm{~F}$ & 5 & $\mathrm{O}$ & \multirow[t]{3}{*}{51} & \multirow[t]{3}{*}{133} \\
\hline & & & & Poor appetite & $\mathrm{R}$ & $\mathrm{F}$ & 5 & $\mathrm{O}$ & & \\
\hline & & & & Sudden diarrhoea & $\mathrm{Fr}, \mathrm{R}$ & F/D & 5 & O & & \\
\hline \multirow[t]{2}{*}{ Hypericum quartinianum A. Rich. } & \multirow[t]{2}{*}{ Hypericaceae } & \multirow[t]{2}{*}{ Ameja } & \multirow[t]{2}{*}{ S } & Snake bite & $\mathrm{R}$ & $\mathrm{F}$ & 5 & $\mathrm{O}$ & & \multirow[t]{2}{*}{889} \\
\hline & & & & Minor bleeding & R & $\mathrm{F}$ & 4 & $\mathrm{O}$ & & \\
\hline \multirow[t]{2}{*}{ Hypoestes aristata (Vahl.) Soland. } & \multirow[t]{2}{*}{ Acanthaceae } & \multirow[t]{2}{*}{ Telenj } & \multirow[t]{2}{*}{$\mathrm{H}$} & Wound & L & $\mathrm{F}$ & 3 & $\mathrm{O}$ & & \multirow[t]{2}{*}{599} \\
\hline & & & & Minor bleeding & L & $\mathrm{F}$ & 3 & O & & \\
\hline \multirow[t]{3}{*}{ Inula confertiflora A. Rich. ${ }^{* *}$} & \multirow[t]{3}{*}{ Asteraceae } & \multirow[t]{3}{*}{ Woynagift } & S & Lumpy skin disease & L & $\mathrm{F}$ & 5 & $\mathrm{O}$ & & 877 \\
\hline & & & & Mange & R & $\mathrm{F}$ & 4 & O & & \\
\hline & & & & Ringworm & R & $\mathrm{F}$ & 4 & O & & \\
\hline Juniperus procera L. & Cupressaceae & Yeabesha Tsid & $\mathrm{T}$ & $\begin{array}{l}\text { Retained placenta/fetal } \\
\text { membrane }\end{array}$ & $\mathrm{R}$ & $\mathrm{F}$ & 5 & O & & 874 \\
\hline Leonotis ocymifolia (Burm.f.) Iwarsson & Lamiaceae & Ras Kimir & S & Anthrax & $\mathrm{R}$ & $\mathrm{F}$ & 5 & O & 26,27 & 871 \\
\hline & & & & Blackleg & L & $\mathrm{F}$ & 5 & $\mathrm{O}$ & & \\
\hline Maesa lanceolata Forssk. & Myrsinaceae & Kelewa & S & Mange & $\mathrm{Fr}$ & $\mathrm{F}$ & 4 & $\mathrm{O}$ & & 42 \\
\hline & & & & Tick infestation & L & $\mathrm{F}$ & 4 & $\mathrm{O}$ & & \\
\hline & & & & Dermatophilosis & $\mathrm{Fr}$ & $\mathrm{F}$ & 2 & O & & \\
\hline & & & & Helminthiasis & $\mathrm{Fr}$ & $\mathrm{F}$ & 5 & $\mathrm{O}$ & & \\
\hline & & & & Parasitic Leech & $\mathrm{Fr}$ & $\mathrm{F}$ & 5 & O & & \\
\hline Nuxia congesta R. Br. ex Fresen. & Loganiaceae & Askwar & $\mathrm{T}$ & Calf pneumonia & L & $\mathrm{F}$ & 5 & $\mathrm{O}$ & & 897 \\
\hline & & & & Cowdriosis & SB & $\mathrm{F}$ & 5 & $\mathrm{O}$ & & \\
\hline & & & & CBPP & SB & $\mathrm{F}$ & 5 & $\mathrm{O}$ & 9 & \\
\hline Ocimum lamiifolium Hochst. & Lamiaceae & Dama Kessie & S & Bloat & L & $\mathrm{F}$ & 5 & O & & 864 \\
\hline & & & & Mucal diarrhoea & L & $\mathrm{F}$ & 5 & $\mathrm{O}$ & & \\
\hline & & & & Poor appetite & L & $\mathrm{F}$ & 5 & $\mathrm{O}$ & & \\
\hline
\end{tabular}


Table 1 List of ethnoveterinary medicinal plants used for treatment of livestock ailments: scientific name; family; local name; growth form; ailment treated; plant parts used; condition of plant part uses; methods of preparation and application, route of administration, plant part mixed with and voucher number (Continued)

\begin{tabular}{|c|c|c|c|c|c|c|c|c|c|c|}
\hline & & & & Bloody diarrhoea & $\mathrm{L}$ & $\mathrm{F}$ & 5 & $\mathrm{O}$ & & \\
\hline \multirow[t]{3}{*}{$\begin{array}{l}\text { Olea europaea L. subsp. cuspidata } \\
\text { (Wall. ex G.Don) }\end{array}$} & Oleaceae & Woyra & $\mathrm{T}$ & Mange & St & $\mathrm{F}$ & 4 & $\mathrm{O}$ & 21,41 & 19 \\
\hline & & & & Ringworm & $\mathrm{R}$ & $\mathrm{F}$ & 2 & $\mathrm{O}$ & & \\
\hline & & & & Lumpy skin disease & $\mathrm{R}$ & $\mathrm{F}$ & 2 & $\mathrm{O}$ & & \\
\hline \multirow[t]{2}{*}{ Pavetta abyssinica Fresen. } & Rubiaceae & Seged achawach & $S$ & Blackleg & SB & $\mathrm{F}$ & 5 & $\mathrm{O}$ & & 485 \\
\hline & & & & Broken limb in calf & SB & $\mathrm{F}$ & 3 & $\mathrm{O}$ & & \\
\hline Pentas lanceolata (Forssk.) Defiers. & Rubiaceae & Yejib Mirkuz & S & Anthrax & R & $\mathrm{F}$ & 5 & $\mathrm{O}$ & 30 & 213 \\
\hline \multirow[t]{5}{*}{ Phytolacca dodecandra L' Herit. } & Phytolaccaceae & Indod & S & Parasitic leech & $\mathrm{L}$ & $\mathrm{F}$ & 5 & $\mathrm{O}$ & & 243 \\
\hline & & & & Mange & $\mathrm{R}$ & $\mathrm{F}$ & 2 & $\mathrm{O}$ & & \\
\hline & & & & Helminthiasis & R & $\mathrm{F}$ & 5 & $\mathrm{O}$ & & \\
\hline & & & & Lice infestation in chicken & $\mathrm{L}$ & $\mathrm{F}$ & 4 & $\mathrm{O}$ & & \\
\hline & & & & Lumpy skin disease & $\mathrm{R}$ & $\mathrm{F}$ & 2 & $\mathrm{O}$ & & \\
\hline Plantago lanceolata $\mathrm{L}$. & Plantaginaceae & Wusha milas tinishu & $\mathrm{H}$ & Rabies & $\mathrm{R}$ & $\mathrm{F}$ & 5 & $\mathrm{O}$ & & 860 \\
\hline Plantago palmata Hook.f. & Plantaginaceae & Wusha milas tiliku & $\mathrm{H}$ & Rabies & $\mathrm{R}$ & $\mathrm{F}$ & 5 & $\mathrm{O}$ & & 895 \\
\hline Podocarpus falcatus (Thunb.) Mirb. & & & & Mange & SB & $\mathrm{F}$ & 2 & $\mathrm{O}$ & & \\
\hline Polyscias fulva (Hiern) Harms & Araliaceae & Yezingero wonber & $\mathrm{T}$ & Minor bleeding & $\mathrm{L}$ & $\mathrm{F}$ & 2 & $\mathrm{O}$ & & 823 \\
\hline Ranunculus multifidus Forssk. & Ranunculaceae & & $\mathrm{H}$ & Dermatophilosis & $\mathrm{R}$ & $\mathrm{F}$ & 4 & $\mathrm{O}$ & & 858 \\
\hline \multirow[t]{4}{*}{ Ricinus communis L. } & Euphorbiaceae & Gulo & S & Mange & $\mathrm{Fr}$ & $\mathrm{F}$ & 4 & $\mathrm{O}$ & 21,33 & 33 \\
\hline & & & & Scabies & $\mathrm{Fr}$ & $\mathrm{F}$ & 4 & $\mathrm{O}$ & & \\
\hline & & & & Ringworm & $\mathrm{Fr}$ & $\mathrm{F}$ & 4 & $\mathrm{O}$ & & \\
\hline & & & & $\begin{array}{l}\text { Retained placenta/fetal } \\
\text { membrane }\end{array}$ & R & $\mathrm{F}$ & 5 & O & & \\
\hline \multirow[t]{4}{*}{ Rubus steudnerii Schweinf. } & Rosaceae & Amoch & S & Bloat & LT & $\mathrm{F}$ & 5 & $\mathrm{O}$ & & 242 \\
\hline & & & & Mucal diarrhoea & $\mathrm{R}$ & $\mathrm{F}$ & 5 & $\mathrm{O}$ & & \\
\hline & & & & Bloody diarrhoea & $\mathrm{R}$ & $\mathrm{F}$ & 5 & $\mathrm{O}$ & 32 & \\
\hline & & & & Blackleg & R & F/D & 5 & $\mathrm{O}$ & & \\
\hline Senecio myriocephalus Sch. Bip. exA. Rich.** & Asteraceae & Sibut & S & Pasteurellosis & $\mathrm{R}$ & $\mathrm{F}$ & 5 & $\mathrm{O}$ & & 891 \\
\hline Sida schimperiana Hochst. ex A. Rich. & Malvaceae & Garda & S & African horse sickness & $\mathrm{R}$ & $\mathrm{F}$ & 5 & O & & 153 \\
\hline
\end{tabular}


Table 1 List of ethnoveterinary medicinal plants used for treatment of livestock ailments: scientific name; family; local name; growth form; ailment treated; plant parts used; condition of plant part uses; methods of preparation and application, route of administration, plant part mixed with and voucher number (Continued)

\begin{tabular}{|c|c|c|c|c|c|c|c|c|c|c|}
\hline Solanecio gigas (Vatke) C. Jeffrey** & Asteraceae & Dengorita & $\mathrm{T}$ & Hepatitis & $\mathrm{L}$ & $\mathrm{F}$ & 5 & $\mathrm{O}$ & & 531 \\
\hline \multirow[t]{2}{*}{ Stephania abyssinica (Dill. \& A. Rich.) Walp. } & Menispermaceae & Yeait hareg & $\mathrm{H}$ & CBPP & $\mathrm{L}$ & $\mathrm{F}$ & 5 & O & & 130 \\
\hline & & & & Calf pneumonia & $\mathrm{L}$ & $\mathrm{F}$ & 5 & O & & \\
\hline $\begin{array}{l}\text { Tephrosia interrupta Hochst. \& Steud ex } \\
\text { Engl. }\end{array}$ & Fabaceae & Gerengere & $S$ & Coccidiosis & $\mathrm{R}$ & $\mathrm{F}$ & 1 & O & 30 & 893 \\
\hline Thalictrum rhynchocarpum Dill. \& Rich. & Ranunculaceae & Sire bizu & $\mathrm{H}$ & African horse sickness & $\mathrm{R}$ & $\mathrm{F}$ & 5 & O & & 535 \\
\hline \multirow[t]{2}{*}{ Trichocladus ellipticus Eckl. \& Zeyh. } & Hamamelidaceae & Abil wuha & $\mathrm{T}$ & Ophthalmic infection & $\mathrm{L}$ & $\mathrm{F}$ & 4 & O & & 896 \\
\hline & & & & African horse sickness & $\mathrm{R}$ & $\mathrm{F}$ & 5 & O & & \\
\hline \multirow[t]{2}{*}{ Triumfetta brachyceras K. Schum. } & Tiliaceae & Leba Giraf & $S$ & Ringworm & $\mathrm{R}$ & F/D & 2 & O & & 886 \\
\hline & & & & Dermatophilosis & $\mathrm{R}$ & $\mathrm{F}$ & 2 & $\mathrm{O}$ & & \\
\hline \multirow[t]{2}{*}{ Vernonia amygdalina Del. } & Asteraceae & Girawa & $\mathrm{T}$ & $\begin{array}{l}\text { Retained placenta/fetal } \\
\text { membrane }\end{array}$ & $\mathrm{R}$ & $\mathrm{F}$ & 5 & $\mathrm{O}$ & & 22 \\
\hline & & & & CBPP & $\mathrm{L}$ & $\mathrm{F}$ & 5 & $\mathrm{O}$ & & \\
\hline Withania somnifera (L.) Dun. & Solanaceae & Gizewa & S & Blackleg & $\mathrm{R}$ & F/D & 5 & O & & 206 \\
\hline \multirow[t]{4}{*}{ Zingiber officinale Roscoe } & Zingiberaceae & Zingibil & $\mathrm{H}$ & Bloat & $\mathrm{Rh}$ & F/D & 5 & O & & \\
\hline & & & & Bloody diarrhoea & $\mathrm{Rh}$ & F/D & 5 & $\mathrm{O}$ & 22 & 850 \\
\hline & & & & Poor appetite & $\mathrm{Rh}$ & F/D & 5 & O & & \\
\hline & & & & Mucal diarrhoea & $\mathrm{Rh}$ & $\mathrm{F} / \mathrm{D}$ & 5 & 0 & & \\
\hline
\end{tabular}

Key: Growth form Tree (T); Shrub (S); Herb (H); Climber (C). Part used (Leaf, L; Root, R; Stem wood, St; Fruit, Fr; Bark, B; Root bark, RB; Stem bark, SB; Flower, Fl; Bulb, Bu; Rhizome, Rh; Latex, Lat). Conditions of part used (CPU) (Dry, D; Fresh, F). Methods of preparation and application (MPAP), 1. Boil, keep to cool for some time and let the animal drink the decoction; 2 . Grind and cover surface of diseased parts with the powder; 3 . Grind and paste the crushed part 4. Extract the juice/oil/latex and pour or paint it; 5. Pound, homogenize with cold water and allow the animal to drink it or pour it on infected part. Route of Administration; (RA) (Oral, O; Dermal, De; Nasal, Na; Optical, Op).

*Endemic species. 


\section{Ethnoveterinary plant knowledge of the people}

The average number of medicinal plants reported by male informants $(4.23 \pm 0.13)$ was found to be higher than that of female respondents $(3.85 \pm 0.19)$, although the difference was statistically non-significant $(\mathrm{P}>0.05)$. Older informants (40-80 years old) who are senior members of the community reported significantly $(\mathrm{P}<0.05)$ higher numbers of medicinal plants than young to middle aged members. Similarly, there was a significant difference $(\mathrm{P}<0.05)$ in the number of medicinal plants reported by key informants and general respondents. Illiterate members of the community knew significantly more medicinal plants than literate ones (Table 2).

\section{Types of livestock ailments and traditional}

\section{diagnosis methods}

A total of 33 veterinary ailment types were identified in the study area for which informants reported to use one or more medicinal plant species to treat specific livestock ailment type (Table 1). A majority of (18\%, 6 ailment types) veterinary ailments claimed by the community belong to gastro-intestinal disease categories, followed by dermatological $(15 \%, 5)$, and parasitic and respiratory (12\% each, 4) disease categories. Diarrhoea and Mange were found to be the most commonly reported forms of veterinary ailments in the District.

Traditional medicine practitioners diagnose veterinary ailments based on observation and/or information obtained by interviewing the livestock owner about major symptoms shown by the diseased animal. Ethnoveterinary herbal remedies are commonly prescribed only after the diseased animal is visually examined by a traditional healer for any symptom on its mouth and foot parts, throat, eyes, nose, ear and/or skin as well as through presence and status of sores/wounds or any form of infection. Rubbing and pasting ethnoveterinary herbal preparations were the commonly reported treatment methods for handling dermatological diseases, whereas oral administration of homogenized herbal preparations was reported as the main treatment method for internal ailments.

\section{Applications of ethnoveterinary remedies}

Although ethnoveterinary medicinal plants of the District are claimed to be applied for ailments affecting cattle, sheep, goats, equines, camels or chicken, the majority of the reported medicinal plants (92\%, 47 species) were found to be applied to treat one or more of the thirty different cattle ailments (Table 1). A relatively few number of ethnomedicinal species (10\%, 5 species) was mentioned to be used against chicken ailments (Figure 2).

Most ethnoveterinary medications (86\%) were reported to comprise remedial parts of a single medicinal plant. However, 14\% were prepared using formulations from two or more medicinal plant species. Amongst all plants reported, the highest proportion of species was claimed to treat diarrhoea (24\%), mange (20\%), ringworm and black leg (16\% each) and bloat (14\%). The highest number of multiple ethnoveterinary uses were recorded for Allium sativum (used against 8 ailment types) and Croton macrostachyus (7 ailment types) (Table 1).

\section{Plant parts used for remedy preparation}

Although different plant parts are reported to be used for remedy preparation by the community, a majority (31\%) of preparations was found to be from root parts alone, followed by mixtures of leaves and roots (21\%) and leaves alone (18\%) (Figure 3). Plants in which roots (65\%, 33 species) and leaves (43\%, 22 species) are utilized as ingredient either alone or mixed with other plant parts were frequent in the medicinal flora of the District. Freshly harvested plant parts were the dominant ones $(83 \%)$ used in remedy preparation whereas the remaining $17 \%$ of remedies were reported to be prepared both from dried or fresh parts of medicinal plant species.

Table 2 Statistical test of significance on average number of reported medicinal plants among different informant groups in Ankober District

\begin{tabular}{|c|c|c|c|c|c|}
\hline Parameters & Informant groups & $\mathbf{N}$ & Average \pm SD & t -value ${ }^{* *}$ & $p$-value \\
\hline \multirow[t]{2}{*}{ Gender } & Male & 235 & $4.23 \pm 0.13$ & 1.61 & 0.1075 \\
\hline & Female & 117 & $3.85 \pm 0.19$ & & \\
\hline \multirow[t]{2}{*}{ Age } & Young members & 122 & $2.59 \pm 0.08$ & -11.65 & $0.0001^{*}$ \\
\hline & Senior members & 230 & $4.90 \pm 0.13$ & & \\
\hline \multirow[t]{2}{*}{ Literacy level } & Illiterate & 85 & $4.73 \pm 0.11$ & -11.90 & $0.0001^{*}$ \\
\hline & Literate & 267 & $2.12 \pm 0.10$ & & \\
\hline \multirow[t]{2}{*}{ Experience (Informant category) } & Key/knowledgeable & 88 & $6.94 \pm 0.16$ & 23.88 & $0.0001^{*}$ \\
\hline & General informant & 264 & $3.16 \pm 0.07$ & & \\
\hline
\end{tabular}

*Significant difference $(p<0.05) ;{ }^{* *} t(0.05)$ (two tailed), $d f=350 ; N=$ Number of respondents. 


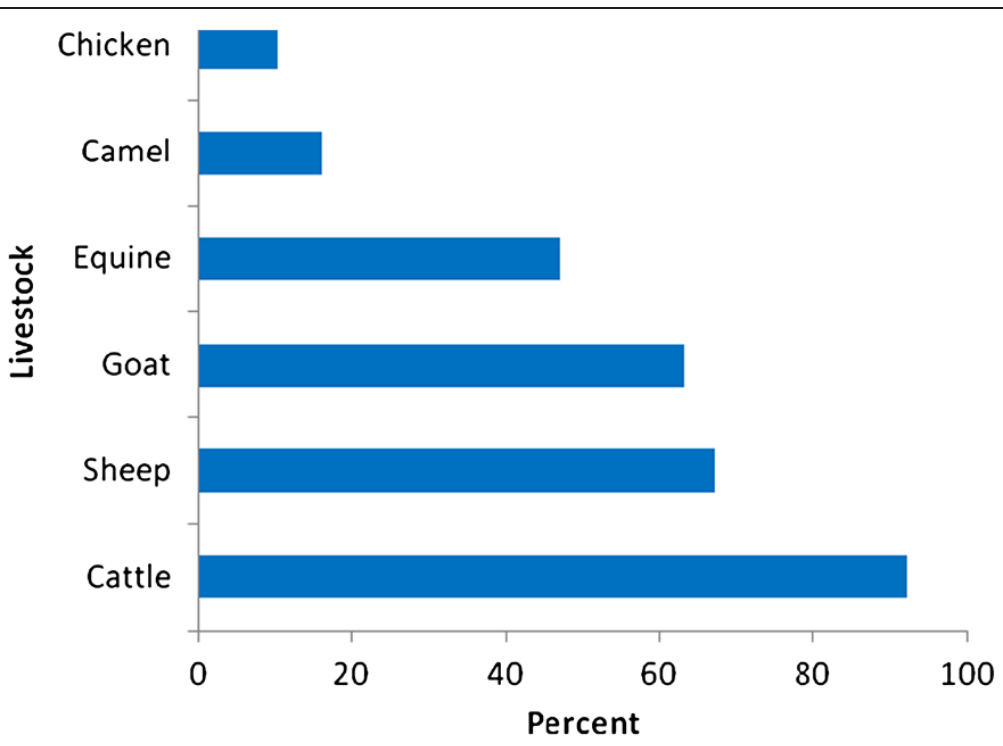

Figure 2 Percentage of ethnoveterinary medicinal plants used for different livestock types in Ankober District.

Modes of remedy preparation, routes of administration and dosages

Various modes of remedy preparation were reported to be used in the District based on type and degree of complexity of livestock ailment. Pounding the remedial part and homogenizing it with cold water was found to be the major mode of remedy preparation (54\%), followed by extracting juice, oil or latex from the plant $(20 \%)$ (Figure 4).

Traditional plant remedies were reported to be administered through oral, dermal, nasal or optical routes of the diseased animal. Oral application was the best-represented route of administration (69 preparations, $53 \%$ ), followed by dermal $(53,40 \%)$, nasal $(6,5 \%)$ and optical $(3,2 \%)$ routes.

Physical appearance of the diseased animal and visually confirmed degree of complexity of illness were used to determine preparation doses to treat livestock ailments. Some traditional practitioners reported use of plastic jugs, glasses, bottles, cups or spoons to determine dosage for some medicinal preparations while others reported to use a handful or finger-sized preparation to treat ailments. However, no strictly standardised doses

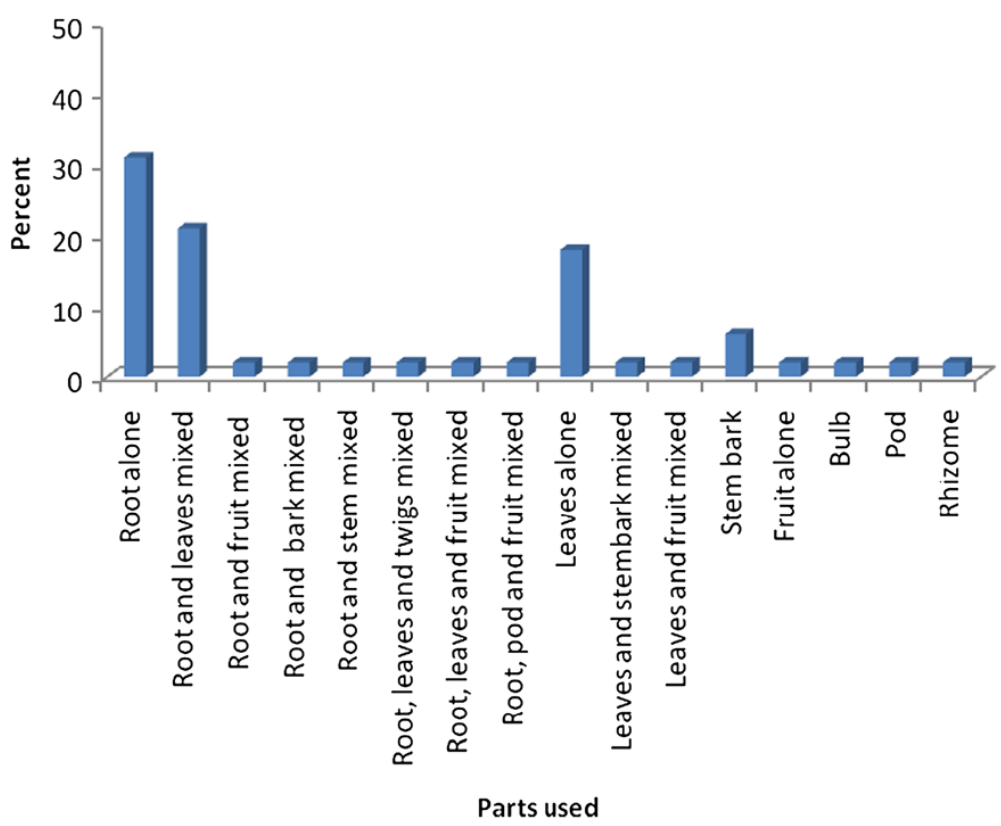

Figure 3 Plant parts used for remedy preparation in Ankober District. 


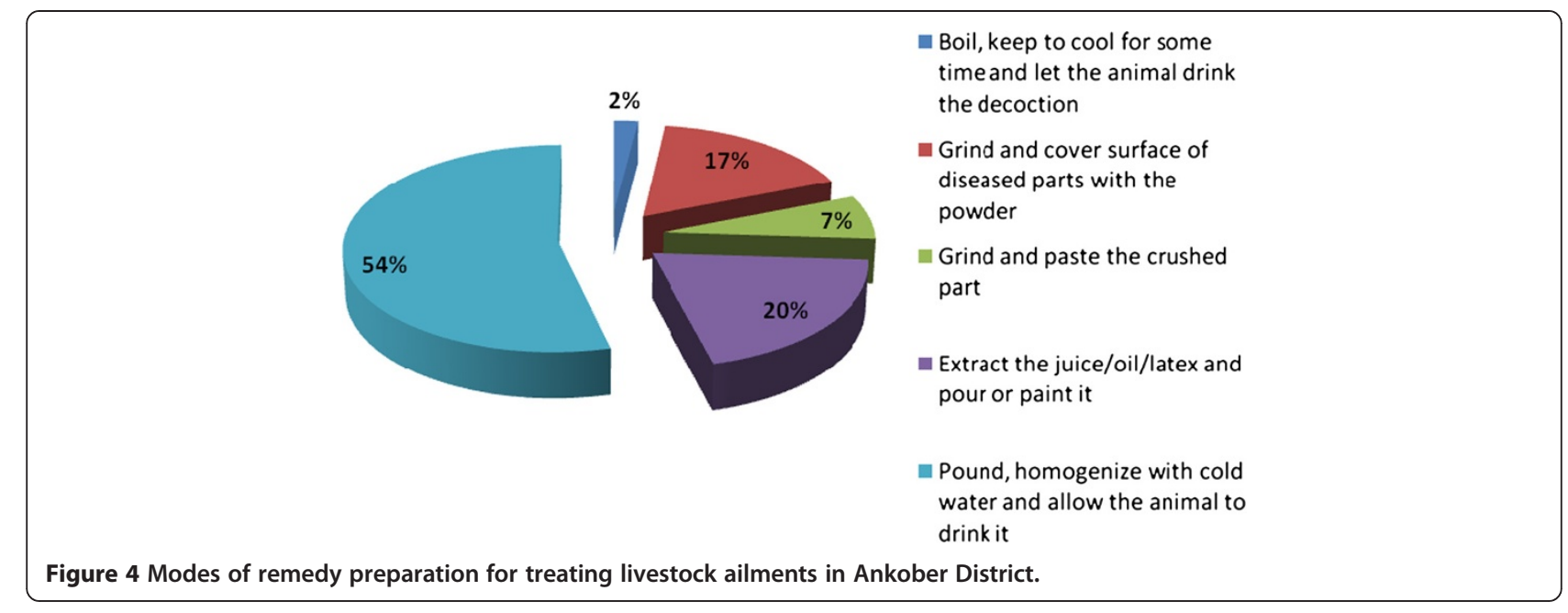

of herbal preparations as known for modern veterinary medicine were reported by traditional healers for any of the preparations used to treat livestock ailments in Ankober District.

\section{Marketability of medicinal plants of ethnoveterinary importance}

About 20\% (10 species) of ethnoveterinary plants were reported to be marketable and could be accessed through purchase at Aliuamba, Derefo, Gorebella, Gorgo, Haramba and Zego local markets in the District. However, out of ten reportedly marketable species only 2, i.e., Embelia schimperi Vatke and Withania somnifera (L.) Dun seem to be purchased utterly for the purpose of their traditional medical applications according to customer explanations. The remaining eight plants mentioned are mainly sold for their non-medicinal uses, but also applied as medicine when the need arises. This means that these eight species are frequently used for non-veterinary purposes but infrequently used for veterinary purposes. The average price of a bunch $(\approx 250-325 \mathrm{~g})$ of the root material of Withania somnifera at five local markets of the District was 3.50 Birr (0.2 USD), whereas the price for a cupful of the fruits of Embelia schimperi was 5.50 Eth Birr (0.3 USD).

\section{Test of efficacy of ethnoveterinary medicinal plants}

Nine major livestock ailment categories were identified from the total 33 veterinary diseases reported in the District. Highest Informants' Consensus Factor (ICF) values were recorded for gastro-intestinal (0.71), ecto and endoparasitic (0.69) and dermatological disease (0.66) categories (Table 3). In addition, highest plant use citation (25.95\%) was recorded for dermatological diseases.

\section{Relative healing potential of medicinal plants}

Embelia schimperi Vatke showed highest fidelity level value (90\%) for gastrointestinal disease category followed by Rubus steudnerii Schweinf. (84\%). Under the dermatological therapeutic category, highest fidelity level value was recorded for Croton macrostachyus Del. (83\%). Achyranthes aspera L. (81\%) has also showed relatively high healing potential record under the external injuries, bleeding and poisoning disease category (Table 4).

\section{Preference ranking of ethnoveterinary plants}

Preference ranking exercise with 15 of the key informants (selected randomly) for medicinal plants that were reported to be used against diarrhoea, the most frequently reported livestock disease under the gastro-intestinal disease category, showed that Embelia schimperi Vatke and Rubus steudnerii Schweinf. were most-preferred species to treat the reported disease (Table 5).

\section{Discussion and conclusion}

Indigenous use and diversity of ethnoveterinary plants The indigenous people of Ankober District rely on livestock as a major support to their livelihoods, employment, crop production, transport and for generating revenue to sustain life. Understanding indigenous knowledge, attitudes and practices of traditional communities about occurrence, treatment, prevention, control and local importance of different livestock ailments and traditionally used medicinal plants against respective ailments is crucial to design and implement meaningful animal health improvement and production strategies [37]. According to Pieroni and co investigators [16], ethnoveterinary studies have long-term output of developing eco-sustainable projects with a primary goal of using plant-based remedies in traditional and also new agricultural and animal breeding systems. Results of this investigation show that people in the District have age-old indigenous knowledge on the use of plants in the wild to treat various livestock ailments. The deep-rooted culture of plant use for successive generations might have played 
Table 3 ICF values of traditional medicinal plants for treating livestock ailments in Ankober

\begin{tabular}{|c|c|c|c|c|c|c|}
\hline No & Disease category & Species & $\%$ all species & Use citations & $\%$ all use citations & ICF \\
\hline 1 & Gastro-intestinal & 7 & 13.72 & 22 & 16.79 & 0.71 \\
\hline 2 & Ecto and endo parasitic & 5 & 9.8 & 14 & 10.68 & 0.69 \\
\hline 3 & Dermatological & 12 & 23.5 & 34 & 25.95 & 0.66 \\
\hline 4 & Sensorial & 3 & 5.88 & 5 & 3.81 & 0.50 \\
\hline 5 & Respiratory & 7 & 13.72 & 11 & 8.39 & 0.40 \\
\hline 6 & External injuries, bleeding and poisoning & 11 & 21.56 & 18 & 13.74 & 0.40 \\
\hline 7 & Reproductive & 3 & 5.88 & 4 & 3.05 & 0.30 \\
\hline 8 & Musculo-skeletal & 8 & 15.68 & 9 & 6.87 & 0.13 \\
\hline 9 & Others & 12 & 23.50 & 14 & 10.68 & 0.20 \\
\hline
\end{tabular}

the role for a sentimental adherence of the community to ancestral medical traditions which are still held as highly valued heritage of the society.

One of the driving factors which made the people of Ankober District to rely on wild plants of ethnoveterinary importance to treat veterinary ailments is inadequate number of formal veterinary clinics (only five) and veterinarians (only eight) available in the area that would never be enough to provide healthcare services for more than 200,000 livestock population [46]. Moreover, almost all of the rural community lives in marginal areas which are not easily accessible to the rare modern veterinary services which are also known for their scorching prices totally unaffordable to the less economically endowed people living there.

Identified ethnoveterinary medicinal flora of Ankober showed that the District is rich in its ethnoveterinary plant diversity and indigenous knowledge associated with each traditionally used species. Comparison of the number and diversity of ethnoveterinary medicinal plant species used in Ankober with other ethnoveterinary research results of cultural communities in Ethiopia [7,40,42,43,63] and elsewhere in Uganda [64], Kenya [15], South Africa [65], Pakistan [66] and India $[67,68]$ confirms the richness of the area in diversity of ethnoveterinary plants. The millennia-old interaction of indigenous people in the area with locally available medicinal plants might have enabled them to develop an indigenous knowledge system best fit to select and use diverse curative medicinal plants to treat frequently occurring livestock diseases. According to Rindos [69], knowledge on plant use is the result of many years of human interaction and selection on the most desirable and successful plants present in the immediate environment at a given time.

Some ethnoveterinary species of Ankober are also found with use reports in other ethno-linguistic communities in the country. Examples are Calotropis procera (Ait.) Aitf and Withania somnifera (L.) Dun. that also occur in the ethnoveterinary medicinal flora of the Afar people [43]; Croton macrostachyus Del., and Ricinus communis L. recorded for the Gilgel Ghibe area and Borana pastoralists [7,44]; and Calpurnia aurea (Ait.) Benth. and Achyranthes aspera L. reported for TanquaAbergele and Kolla-Tembien Districts [63]. Similarity of medicinal plant species used in different communities can be attributed to cross-fertilization of cultural knowledge among different ethno-linguistic groups besides distribution/availability of the species in use in areas investigated for their ethnomedicinal knowledge.

Best-representation of ethnoveterinary species from families Asteraceae, Asclepiadaceae, Euphorbiaceae and

Table 4 Fidelity level value of medicinal plants commonly reported against a given veterinary ailment category

\begin{tabular}{|c|c|c|c|c|c|}
\hline No & Medicinal plant & Therapeutic category & IP & IU & FL Value $\%$ \\
\hline 1 & Embelia schimperi Vatke & Gastro-intestinal & 36 & 40 & 90 \\
\hline 2 & Rubus steudnerii Schweinf. & Gastro-intestinal & 26 & 31 & 84 \\
\hline 3 & Croton macrostachyus Del. & Dermatological & 25 & 30 & 83 \\
\hline 4 & Achyranthes aspera L. & External injuries, bleeding and poisoning & 39 & 48 & 81 \\
\hline 5 & Phytolacca dodecandra L' Herit. & Ecto and endo-parasites & 23 & 29 & 79 \\
\hline 6 & Cissampelos mucronata A. Rich. & Respiratory & 24 & 32 & 75 \\
\hline 7 & Trichocladus ellipticus Eckl. \& Zeyh. & Sensorial & 19 & 27 & 70 \\
\hline 8 & Withania somnifera (L.) Dun. & Musculo-skeletal & 28 & 39 & 71 \\
\hline 9 & Aeonium leucoblepharum Webb ex A. Richard & Reproductive & 17 & 23 & 74 \\
\hline 10 & Ocimum lamiifoium Hochst. & Gastro-intestinal & 30 & 38 & 66 \\
\hline
\end{tabular}


Table 5 Results of preference ranking of medicinal plants reported for treating livestock Diarrhoea

\begin{tabular}{|c|c|c|c|c|c|c|c|c|c|c|c|c|c|c|c|c|c|}
\hline \multirow[t]{2}{*}{ Medicinal plants for diarrhoea } & \multicolumn{17}{|c|}{ Informants labelled $A$ to $O$} \\
\hline & A & B & $\mathrm{C}$ & D & $\mathbf{E}$ & $\mathrm{F}$ & G & H & 1 & $J$ & $\mathrm{~K}$ & $\mathbf{L}$ & M & $\mathrm{N}$ & 0 & Total score & Rank \\
\hline Dodonea angustifolia L. f. & 1 & 3 & 3 & 2 & 2 & 3 & 1 & 2 & 3 & 4 & 2 & 3 & 4 & 7 & 7 & 47 & 5 \\
\hline Gomphocarpus fruticosus (L.) Ait. F. & 2 & 1 & 2 & 3 & 1 & 1 & 5 & 3 & 1 & 3 & 1 & 4 & 2 & 3 & 2 & 34 & 6 \\
\hline Zingiber officinale Roscoe & 6 & 7 & 6 & 5 & 5 & 6 & 4 & 4 & 5 & 7 & 5 & 7 & 6 & 4 & 4 & 81 & 3 \\
\hline Clutia abyssinica Jaub. \& Spach & 3 & 2 & 1 & 1 & 3 & 4 & 2 & 1 & 2 & 2 & 3 & 2 & 3 & 1 & 1 & 31 & 7 \\
\hline Ocimum lamiifolium Hochst. & 4 & 4 & 4 & 6 & 4 & 2 & 3 & 6 & 7 & 1 & 4 & 1 & 1 & 2 & 3 & 52 & 4 \\
\hline Rubus steudnerii Schweinf. & 5 & 6 & 5 & 7 & 6 & 5 & 7 & 5 & 4 & 5 & 6 & 5 & 5 & 5 & 6 & 82 & 2 \\
\hline Embelia schimperi Vatke & 7 & 5 & 7 & 4 & 7 & 7 & 6 & 7 & 6 & 6 & 7 & 6 & 7 & 6 & 5 & 93 & 1 \\
\hline
\end{tabular}

N:B-Scores in the table indicate ranks given to medicinal plants based on their efficacy. Highest number (7) for the medicinal plant which informants thought was most effective in treating diarrhoea and the lowest number (1) for the least-effective plant.

Ranunculaceae could be related to their wider distribution and use in Ethiopia/the flora area [55,56,58,61]. These families were also reported to have the largest share of ethnomedicinal species in other ethnobotanical inventories $[42,43,63,70]$.

\section{Growth habit, source and remedial parts of Ethnoveterinary plants}

Results also showed most-frequent utilization of shrubs followed by herbs in remedy preparation. Dominance of shrubs was also reported by earlier ethnobotanical inventories [30,71-73]. In contrast, other investigators [64,70,74-76] reported dominance of herbaceous species for ethnomedicinal preparation in Ethiopia and elsewhere. However, the variation in dominance of growth forms of medicinal plants used among different ethnolinguistic groups in the country could be attributed to the wide agro-ecological diversity and specific indigenous knowledge of different communities. Results also evidenced the dominant practice of harvesting majority (80\%) of ethnoveterinary plants of Ankober from noncultivated sources. This would indicate the extent of anthropogenic pressure exerted on wild plant resources of the area. Overdependence on wild resources coupled with shrinking of the wild habitat due to ever-increasing population pressure pose a threat to medicinal plant wealth of the area. Comparable trends in overharvesting medicinal plants from the wild were also reported [30,44,64,77-79].

The finding of roots to be the most harvested plant parts used for ethnoveterinary remedy preparation in the District might be associated with traditional beliefs in different communities about a powerful therapeutic effect of root parts for treating various ailments [80-82]. However, harvesting roots for remedy preparation is always accompanied with complete removal of the respective medicinal plant from the natural environment has been observed in many cases posing challenges by affecting eventual survival of the individual and ultimately the species. According to Sheldon et al. [83], the main factor to be considered for conservation and sustainable use of medicinal plants is the particular plant part harvested for its curative value and the way it is harvested. Although leaves were also reported to be harvested from many ethnoveterinary plants in Ankober, their harvesting could be regarded as sustainable since some leaves are left on the parent plant to carry on its life activities. Overharvesting of roots for remedy preparation was also reported for different cultural groups in the country and elsewhere [30,31,64,70,78,84] calling for a coordinated conservation measure built on local sustainable harvesting methods like not removing the major roots and covering the expose parts to save the fast eroding therapeutic plants ruthlessly hunted for their roots. Results also indicate pronounced preference of traditional healers in Ankober to make use of freshly harvested plant parts (83\%) for remedy preparation over dried forms. This could be attributed to the wide-spread traditional belief of attaining high efficacy from fresh remedies due to higher presence of active ingredients in the form of secondary metabolites in the cases of fresh plant parts which community members rightly thought could be lost on drying. Similar observations were reported $[30,70]$ for other cultural groups living in Ethiopia.

\section{Ethnoveterinary plant knowledge of the community}

Comparison of medicinal plant knowledge held among community members of varying age groups in Ankober showed a significant difference $(\mathrm{P}<0.05)$ in plant use by senior/elderly members of the community $(4.90 \pm 0.13)$ over younger ones $(2.59 \pm 0.08)$. This could be related to a higher degree of cultural contact and experience of the elderly members with curative plants than that of younger members in the community as described by Silva and co-workers [85]. Modernization and high degree of secrecy on passing knowledge on medicinal plants within the family circle only to elder sons, and lack of interest on traditional remedies by younger groups might also explain the decline of indigenous knowledge 
going down the generation ladder in the District. Ethnobotanical inventories in Ethiopia [30,40,79,84,86] and elsewhere in other countries $[85,87,88]$ also share a similar concern on the knowledge gap down generations in different cultural groups. Systematic documentation of indigenous knowledge on medicinal plant use through ethnobotanical inventories is essential to safeguard such fast-eroding knowledge among successive generations. The difference in knowledge gap observed among key and general informants in this investigation could also be associated with high degree of experience/specialisation and greater contact with therapeutic plants in the first than the latter group [89]. Results showed that both men and women members of the community in Ankober are knowledgeable on medicinal plant use despite the relative dominance of medicinal plant tradition by men which could be associated with the traditional flow of information along the male line in the country [84].

\section{Livestock ailments, remedy preparation and traditional diagnosis methods}

The observed frequent utilization of finely pounded remedial parts which are then homogenized with cold water (54\%) and used against various ailments could relate to age- old traditional experiences on proven efficacy of such products. Homogenizing remedial parts with water has also been reported from other cultural groups $[30,70,80]$ against various ailments. The observed trend of using two or more medicinal plants (accounting for about 14\% of preparations) for treating livestock ailments may be attributed to the expected synergistic effect of combinations of parts and their bioactive ingredients to treat ailments. Giday et al. [90] have also reported therapeutic efficacy of combinations of medicinal plant parts used in Shinasha, Agew-awi and Amhara peoples living in northwest Ethiopia for treating various ailments.

Identification of specific livestock ailment types in the area was found to be made based on age-old cultural knowledge on symptoms and corresponding livestock illnesses held in the memories of indigenous people. The same was found true in selecting curative plants which were thought to be most appropriate to heal different veterinary diseases. However, no standardised volume or weight measurements were set by traditional healers on the amount of herbal prescriptions for different livestock ailments. Ethnoveterinary studies conducted in Pakistan [91] and Brazil [20] have also reported the lack of standardised doses in traditional prescriptions of livestock remedies. In addition, similar patterns of diagnosis and herbal prescriptions were reported [30,70,71,73-75]. According to Abebe [92], lack of precision and standardization has been cited as one of the most important shortcomings of the traditional healthcare system in Ethiopia.
The observed diverse medicinal uses of plants in the area against various livestock ailments indicates presence of inherent curative property engraved within each medicinal plant which still plays an essential role for the production of relatively healthier cattle, sheep, goats, equines, camels or chicken. The relatively high number of medicinal plants cited for treating cattle ailments may also be related to incidence of more diseases affecting cattle populations in the area. Similarly, the highest proportion of medicinal plants used to treat dermatological diseases in the study area could also be related to high incidence of such diseases in Ankober. This was justified by presence of highest number of dermatological diseases (accounting 15\% of livestock ailments in Ankober District) reported by community members. In addition, the finding indicates that traditional veterinary practices in the area are well focussed to the most prevalent health problems. Presence of largest share of ethnoveterinary plants for treating cattle ailments was also reported for other cultural groups in the country [39].

\section{Marketability of ethnoveterinary plants}

Local communities in Ankober mainly collect and use ethnoveterinary plants for a private use with their own livestock. However, a limited scale of buying and selling traditional medicine was observed at Aliuamba, Derefo, Gorebella, Gorgo, Haramba and Zego local markets in the District. Though 20\% (10 species) of ethnoveterinary medicinal plants were shown to be available on the market only 2 species i.e., Embelia schimperi (0.3 USD per cup of fruits) and Withania somnifera (0.2 USD per bunch of root material) seem to be purchased solely for use in traditional medical applications. Market price of these medicinal plants mainly depends on the distance travelled to collect remedial plants and availability of species in the area. Embelia schimperi and Withania somnifera were reported for scarcity in the area by informants and local retailers whereby the latter was explained to be due to overharvesting of species. This made collectors travel long distances to gather these species. The remaining eight marketed species were mainly sold for their uses other than medicine i.e., food, lumbering and firewood purposes though they were mentioned to be occasionally used as remedies when there is a need. Local retailers of medicinal plants, though not herbalists, have also shown some knowledge on use, local distribution and local market demand of economically useful medicinal plants which in turn indicated that knowledge on curative plants is not limited only to local healers or traditional herbalists. Status and availability of marketable medicinal plants were also reported in various sources [93-96] for other cultural groups in Ethiopia. 


\section{Test of efficacy, healing potential and ranking of ethnoveterinary plants}

The observed high informants' consensus ( $\mathrm{ICF}=0.71)$ on ethnoveterinary medicinal plants used to treat gastrointestinal diseases in the area indicated popularity of curative plants against diseases in the latter category. Sharma et al. [22] have explained that high ICF values indicate highest share of similar plant use information within a community. The recorded high plant use citation $(25.95 \%)$ for treating ailments in the dermatological disease category may also indicate the relatively high incidence of such diseases and ease of identifying ailments and corresponding curative plants occurring in the District. Since high ICF values are indicative of selecting target plants for search of bioactive compounds [62], our research team has used the results obtained in this investigation to select 21 elite species for further investigation of their pharmacological properties.

Highest fidelity level values were obtained for Embelia schimperi (90\%) and Rubus steudnerii (84\%) in the gastrointestinal disease category, and Croton macrostachyus (83\%) for the dermatological therapeutic category indicates relatively high healing potential of the species for treating ailments under the respective ailment categories $[62,97]$. Thus, these results would call for pharmacological investigations on these plants since high percentage of informants agreed on their curative values. Hence, plants with high fidelity level value obtained in this investigation are now being tested for pharmacological activities by our research team.

Output of the preference ranking exercise also indicated that Embelia schimperi and Rubus steudnerii are the most-preferred ethnoveterinary medicinal plants used to treat diarrhoea, the most commonly reported disease in the area. This may be attributed to the presence of bioactive compounds against causative agents of diarrhoea in these species. Hence, both species are also being investigated for further antimicrobial activities against different microbial strains by the same research group.

Generally, results of this investigation evidenced a rich ethnoveterinary medicinal plants and indigenous knowledge on their utilization in Ankober District. It is therefore worth engaging in the conservation of these plants which in turn calls for conserving their habitats. This action would save many more plants as well as medicinal plants for humans. Validating bioactivity of ethnoveterinary medicinal plants that are most agreed for their curative role by the community is highly recommended to come up with further scientific evidence which can be used to support the livestock healthcare system in the country and globally in the years ahead.

\section{Competing interest}

The authors declare that they have no competing interests.
Authors' contributions

All authors have equal contribution and all authors have read and approved the final manuscript.

\section{Acknowledgments}

We are indebted to the inhabitants of Ankober District, particularly the traditional medical practitioners who unreservedly shared with us their knowledge on medicinal plants. We are grateful for the Research and Graduate Programs Office, Addis Ababa University (AAU) for funding the cost of field research. Staff members of The National Herbarium (Ethiopia) are also acknowledged for their cooperation with all aspects of the herbarium work. Dr. Henok Lulekal from WDAO, North Shewa Ethiopia, is acknowledged for translating local names of diseases into their English equivalents based on descriptions of symptoms. Staff members of the digital libraries of AAU, UGent and UNECA are also acknowledged for facilitating reference provision for this work. We thank Mr. Ashenafi Mihrete, Mr. Abebe Shewamene, Mr. Getu Shewamene and Mr. Dawit Tewabe for their field assistantship. We also extend our gratitude to administrative officials of Ankober Woreda Development Association, Ankober District, Debre Berhan University and Increasing People Opportunity (IPO) office in Ethiopia for their support in facilitating the study. Fikirte Demisse and Mare Addis are also truly acknowledged for their contribution in developing the digital map of the study area.

\section{Author details}

'Laboratory for Tropical and Subtropical Agriculture and Ethnobotany, Department of Plant Production, Faculty of Bio-Science Engineering, Ghent University, Coupure links 653, 9000 Ghent, Belgium. ${ }^{2}$ Department of Plant Biology and Biodiversity Management, College of Natural sciences, Addis Ababa University, P.O. Box 3434, Addis Ababa, Ethiopia. ${ }^{3}$ Department of Crop Science and Agroforestry, Faculty of Tropical Agrisciences,

Czech University of Life Sciences Prague, Kamycka 129, 16521 Prague 6-Suchdol, Czech Republic

Received: 17 October 2013 Accepted: 26 January 2014

Published: 11 February 2014

\section{References}

1. MOA: Ethiopia Animal Health Year Book (2009/10). Addis Ababa: Federal Ministry of Agriculture Animal and Plant Health Regulatory Directorate (APHRD); 2010.

2. Behnke R, Metaferia F: IGAD Livestock Policy Initiative. Working paper. No 02-11. United Kingdom: Odessa Centre; 2011.

3. USDA: Giving Livestock Feed a Boost in Ethiopia. http://feedthefuture.gov/ article/giving-livestock-feed-boost-ethiopia.

4. Ethiopia Animal Health Year Book: Ethiopia Animal Health Year Book 2011 Addis Ababa, Ethiopia: Ministry of Agriculture; 2011.

5. FAOstat. http://faostat3.fao.org/home/index.html\#DOWNLOAD.

6. Duguma B: Smallholder livestock production and marketing systems in the Haramaya District, Eastern Ethiopia. Basic Res J Agric Sci Rev 2013, 2:122-129.

7. Sori T, Bekana M, Adugna G, Kelbessa E: Medicinal plants in the ethnoveterinary practices of Borana pastoralists, Southern Ethiopia. Int J Appl Res Vet Med 2004, 2:220-225.

8. Admassu B: Primary Animal Healthcare in Ethiopia: The Experience so far. http://sites.tufts.edu/capeipst/files/2011/03/Admassu-Mombasa.pdf.

9. Fromsa A, Jobre $Y$ : Estimated annual economic loss from organ condemnation, decreased carcass weight and milk yield due to bovine hydatidosis (Echinococcus granulosus, Batsch, 1786) in Ethiopia. Ethiop Vet J 2012, 16(2):1-14.

10. Mathias E, McCorkle CM: Animal Health. In Biotechnology: Building on Farmers Knowledge. Edited by Bunders J, Haverkort B, Hiemstra W. London: Macmillan; 1996.

11. Winter K, McClatchey W: Quantifying evolution of cultural interactions with plants: implications for managing diversity for resilience in socialecological systems. Funct Ecosyst Communities 2008, 2(Special issue 1):1-10.

12. Grade JT, Tabuti JRS, Van Damme P: Ethnoveterinary knowledge in pastoral Karamoja, Uganda. J Ethnopharmacol 2009, 122:273-293.

13. Cetinkaya G: Challenges for the maintenance of traditional knowledge in the Satoyama and Satoumi ecosystems, Noto peninsula, Japan. Hum Ecol Rev 2009, 16:27-40. 
14. UNESCO: Safeguarding of the Intangible Cultural Heritage. Paris; 2005. http://portal. unesco.org/culture/en/ev.phpURL_ID=16429\&URL_DO=DO_TOPIC\&URL_ $\mathrm{SECTION}=201 . \mathrm{html}$.

15. Njoroge NG, Bussmann RW: Herbal usage and informant consensus in ethnoveterinary management of cattle diseases among the Kikuyus (Central Kenya). J Ethnopharmacol 2006, 108:332-339.

16. Pieroni A, Giusti ME, De Pasquale C, Lenzarini C, Censorii E, Reyes GonzálesTejero M, Sánchez-Rojas CP, Ramiro-Gutiérrez JM, Skoula M, Johnson C, Sarpaki A, Della A, Paraskeva-Hadijchambi D, Hadjichambis A, Hmamouchi M, S El-Jorhi S, El-Demerdash M, El-Zayat M, Al-Shahaby O, Zahia Houmani Z, Scherazed M: Circum-Mediterranean cultural heritage and medicinal plant uses in traditional animal healthcare: a field survey in eight selected areas within the RUBIA project. J Ethnobiol Ethnomed 2006, 2:16.

17. Lans C, Turner N, Khan T, Brauer G, Boepple W: Ethnoveterinary medicines used for ruminants in British Columbia, Canada. J Ethnobiol Ethnomed 2007, 3:11.

18. McGaw $\sqcup$, Eloff JN: Ethnoveterinary use of southern African plants and scientific evaluation of their medicinal properties. J Ethnopharmacol 2008, 119:559-574.

19. Farooq Z, lqbal Z, Mushtaq S: Ethnoveterinary practices for the treatment of parasitic diseases in livestock in Cholistan desert (Pakistan). J Ethnopharmacol 2008, 118:213-219.

20. Monteiro MVB, Bevilaqua CML, Palha MDC, Braga RR, Schwanke K, Rodrigues ST, Lameira OA: Ethnoveterinary knowledge of the inhabitants of Marajo' Island, Eastern Amazonia, Brazil. Acta Amazon 2011, 41:233-242.

21. Martínez GJ, Luján MC: Medicinal plants used for traditional veterinary in the Sierras de Córdoba (Argentina): an ethnobotanical comparison with human medicinal uses. J Ethnobiol Ethnomed 2011, 7:23.

22. Sharma R, Manhas RK, Magotra R: Ethnoveterinary remedies of disease among milk yielding animals in Kathua, Jammu and Kashmir, India. J Ethnopharmacol 2012, 141:265-272.

23. Offiah NV, Makama S, Elisha IL, Makoshi MS, Gotep JG, Dawurung CJ, Oladipo OO, Lohlum AS, Shamaki D: Ethnobotanical survey of medicinal plants used in the treatment of animal diarrhoea in Plateau State, Nigeria. BMC Vet Res 2011, 7:36.

24. Benítez G, Reyes González-Tejero M, Molero-Mesa J: Knowledge of ethnoveterinary medicine in the Province of Granada, Andalusia, Spain. J Ethnopharmacol 2012, 139:429-439.

25. Pieroni A, Münz H, Akbulut M, Baser KHC, Durmuskahya C: Traditional phytotherapy and trans-cultural pharmacy among Turkish migrants living in Cologne, Germany. J Ethnopharmacol 2005, 102:69-88.

26. Fullas F: Ethiopian medicinal plants in veterinary healthcare. A mini-review. Ethiopian e-Journal for Research and Innovation Foresight 2010, 2:48-58.

27. Abebe D: Traditional medicine in Ethiopia. The attempt being made to promote it for effective and better utilization. SINET: Ethiop J Sci 1986, 9:61-69.

28. Demissew S, Dagne E: Basic and applied research in medicinal plants. In Proceedings of the National Workshop on Biodiversity Conservation and Sustainable Use of Medicinal Plants in Ethiopia: 28 April-1May 1998. Edited by Zewdu M, Demissie A. Addis Ababa, Ethiopia: Institute of Biodiversity Conservation and Research; 2001:29-33.

29. Wabe NT, Mohammed MA, Raju NJ: An ethnobotanical survey of medicinal plants used in the Southeast Ethiopia used in traditional medicine. Spatula DD 2011, 1:153-158.

30. Lulekal E, Kelbessa E, Bekele T, Yineger H: An ethnobotanical study of medicinal plants in Mana Angetu District, southeastern Ethiopia. J Ethnobiol Ethnomed 2008, 4:10.

31. Abebe D, Ayehu A: Medicinal Plants and Enigmatic Health Practices of Northern Ethiopia Addis Ababa. B.S.P.E: Ethiopia; 1993.

32. Lynam T, De Jong W, Sheil D, Kusumanto T, Evans K: A review of tools for incorporating community knowledge, preferences, and values into decision making in natural resources management. Ecol Soc 2007, 12(1):5.

33. Cos P, Vlietinck AJ, Vanden Berghe D, Maes L: Anti-infective potential of natural products: How to develop a stronger in vitro 'proof- of- concept'. J Ethnopharmacol 2006, 106:290-302.

34. Alemu L: Studies on the Traditional Medicinal Plants of Veterinary Importance in and Around Bahir Dar. DVM Thesis. Addis Ababa: Addis Ababa University; 1993.

35. Mesfin T, Obsa T: Ethiopian traditional veterinary practices and their possible contribution to animal production and management. Revue Scientifiaue et Technique De L Office International Des Epizooties 1994, $13: 417-424$.
36. Tolosa A: Ethnoveterinary knowledge in central highlands of Ethiopia, Sheno, North Shoa. In Proceedings of the 11th Conference of the Ethiopian Veterinary Association: June 1997. Edited by Ethiopian Veterinary Association. Addis Ababa, Ethiopia: Ethiopian Veterinary Association; 1997:110-125.

37. Wirtu G, Adugna G, Samuel T, Kelbessa E, Gelato A: Aspects of farmers' knowledge, attitudes and practices of animal health in central Ethiopia. In Proceedings of an International Conference on Ethnoveterinary Medicine, Alternative for Livestock Development: 4-6 November 1997. Edited by Mathias E, Rangnekar DV, McCorkle C. Pune, India: BAIF Development research foundation; 1999:41-52.

38. Mesfin T: Ethnoveterinary Practices of Camel Herders of Southern Afar Area; 2000. http://ossrea.net/nw/ethiopia/nw-02.htm.

39. Giday M, Ameni $G$ : An ethnobotanical survey on plants of veterinary importance in two woredas of Southern Tigray, Northern Ethiopia. SINET: Ethiopian Journal of Science 2003, 26:123-136.

40. Yineger $H$, Yewhalaw D, Teketay D: Ethnomedicinal plant knowledge and practice of the Oromo ethnic group in Southwestern Ethiopia. J Ethnobiol Ethnomed 2008, 4:11

41. Woldegerima B, Abula T, Ragunathan M: Ethnoveterinary medicinal plants in Dabat District, western Ethiopia. Pharmacogn Mag 2008, 4:93-99.

42. Bekele D, Asfaw Z, Petros B, Tekie H: Ethnobotanical study of plants used for protection against insect bite and for the treatment of livestock health problems in rural areas of Akaki District, Eastern Shewa, Ethiopia. Top class Journal of Herbal Medicine 2012, 1:12-24.

43. Giday M, Teklehaymanot T: Ethnobotanicl study of plants used in management of livestock health problems by Afar people of Ada'ar District, Afar Regional State, Ethiopia. J Ethnobiol Ethnomed 2013, 9:8.

44. Yineger $H$, Yewhalaw D, Teketay D: Plants of veterinary importance in Southwestern Ethiopia: the case of Gilgel Ghibe area. Forests, Trees and Livelihoods 2008, 18:165-181.

45. Zatew E, Yemane B: Millenium Ankober. Gorebela: Ankober District, Culture and Tourism Office; 2007.

46. Ankober Woreda Finance, Planning and Economic Development Office: The Socio Economic Profile of Ankober District. Ankober, Gorebela: AWFPO; 2012.

47. Teketay D, Bekele T: Floristic composition of Wof-Washa natural forest, Central Ethiopia: implications for the conservation of biodiversity. Feddes Repertorium 1995, 106:127-147.

48. BirdLife International: Serinus Ankoberensis. IUCN 2012. IUCN Red List of Threatened Species. Version 2012.2. www.iucnredlist.org.

49. Martin GJ: Ethnobotany: A Methods Manual. London, UK: Chapman and Hall; 1995.

50. Davis A, Wagner JR: Who knows? On the importance of identifying "Experts" when researching local ecological knowledge. Hum Ecol 2003, 31:463-489.

51. Cotton CM: Ethnobotany: Principles and Applications Chichester. New York: John Wiley and Sons Ltd.; 1996.

52. Alexiades M: Collecting ethnobotanical data. An introduction to basic concepts and techniques. In Selected Guideline for Ethnobotanical Research: A Field Manual. Edited by Alexiades M, Sheldon JW. Bronx, New York: The New York Botanical Garden; 1996:53-94.

53. Balick MJ, Cox PA: Plants, People and Culture: Science of Ethnobotany. New York: Scientific American Library; 1996.

54. Edwards S, Demissew S, Hedberg I: Flora of Ethiopia and Eritrea. Hydrocharitaceae to Arecaceae Volume 6. The National Herbarium, Addis Ababa, Ethiopia: Department of Systematic Botany, Uppsala, Sweden; 1997

55. Edwards S, Tadesse M, Demissew S, Hedberg I: Flora of Ethiopia and Eritrea. Magnoliaceae to Flacourtiaceae Volume 2. The National Herbarium, Addis Ababa, Ethiopia: Department of Systematic Botany, Uppsala, Sweden; 2000

56. Edwards S, Tadesse M, Hedberg I: Flora of Ethiopia and Eritrea. Canellaceae to Euphorbiaceae Volume 2. Uppsala, Sweden: Department of Systematic Botany; 1995. The National Herbarium, Addis Ababa, Ethiopia.

57. Hedberg I, Edwards S: Flora of Ethiopia and Eritrea. Pittosporaceae to Araliaceae Volume 3. The National Herbarium, Addis Ababa, Ethiopia: Department of Systematic Botany, Uppsala, Sweden; 1989.

58. Hedberg I, Edwards S, Nemomissa S: Flora of Ethiopia and Eritrea. Apiaceae to Dipsacaceae Volume 4. Uppsala, Sweden: Department of Systematic Botany; 2003. The National Herbarium, Addis Ababa, Ethiopia.

59. Hedberg I, Friis I, Edwards S: Flora of Ethiopia and Eritrea. Asteraceae Volume 4 part 2. Uppsala, Sweden: Department of Systematic Botany; 2004. The National Herbarium, Addis Ababa, Ethiopia.

60. Hedberg I, Kelbessa E, Edwards S, Demissew S, Persson E: Flora of Ethiopia and Eritrea. Gentianaceae to Cyclochelicaceae Volume 5. Uppsala, Sweden: 
Department of Systematic Botany; 2006. The National Herbarium, Addis Ababa, Ethiopia.

61. Tadesse M: Flora of Ethiopia and Eritrea, Part 2: Asteraceae (Compositae) Volume 4. Addis Ababa, Ethiopia: The National Herbarium, Addis Ababa University; 2004

62. Heinrich M, Ankli A, Frei B, Weimann C, Sticher O: Medicinal plants in Mexico: healers' consensus and cultural importance. Soc Sci Med 1998, 47:1859-1871

63. Gebrezgabiher G, Kalayou S, Sahle S: An ethno-veterinary survey of medicinal plants in woredas of Tigray region, Northern Ethiopia. Int J Biodivers Conserv 2013, 5:89-97.

64. Tabuti JRS, Lye KA, Dhillion SS: Traditional herbal drugs of Bulamogi, Uganda: plants, use and administration. J Ethnopharmacol 2003, 88:19-44.

65. Botha CJ, Swan GE, Van der Merwe D: Use of ethnoveterinary medicinal plants in cattle by Setswana-speaking people in the Madikwe area of the North West Province of South Africa. J S Afr Vet Assoc 2001, 72:189-196.

66. Khan MA, Khan MA, Hussain M: Ethnoveterinary medicinal uses of plants of Pooch Valley Azad, Kashmir. Pakistan Journal of Weed Science Research 2012, 18:495-507.

67. Selvaraju A, Ayyanar M, Rathinakumar SS, Sekar T: Plants used in ethnoveterinary medicine by malayali tribals in Salem district of Tamil Nadu, India. Med Plants 2011, 3:1-7.

68. Devendrakumar D, Anbazhagan M: Ethnoveterinary medicinal plants used in Perambalur District, Tamil Nadu. Res Plant Biol 2012, 2(3):24-30.

69. Rindos D: The Origins of Agriculture: An Evolutionary Perspective. Cornell University. Orlando: Academic Press, INC; 1984

70. Yineger $\mathrm{H}$, Kelbessa $\mathrm{E}$, Bekele $\mathrm{T}$, Lulekal E: Ethnoveterinary medicinal plants at Bale Mountains National Park, Ethiopia. J Ethnopharmacol 2007, 112:55-70.

71. Bekalo TH, Woodmatas SD, Woldemariam ZA: An ethnobotanical study of medicinal plants used by local people in the lowlands of Konta Special Woreda, southern nations, nationalities and peoples regional state, Ethiopia. J Ethnobiol Ethnomed 2009, 5:26.

72. Hunde D, Asfaw Z, Kelbessa E: Use of traditional medicinal plants by people of 'Boosat' sub district, Central Eastern Ethiopia. Ethiop J Health Sci 2006, 16:141-155.

73. Mesfin F, Demissew S, Tekelaymanot T: An Ethnobotanical study of medicinal plants in Wonago Woreda, SNNPR, Ethiopia. J Ethnobiol Ethnomed 2009, 5:28.

74. Giday M, Asfaw Z, Elmqvist T, Woldu Z: An ethnobotanical study of medicinal plants used by the Zay people in Ethiopia. J Ethnopharmacol 2003, 85:43-52.

75. Giday M, Asfaw Z, Woldu Z: Ethnomedicinal study of plants used in Sheko ethnic group of Ethiopia. J Ethnopharmacol 2010, 132:75-85.

76. Muthu C, Ayyanar M, Raja N, Ignacimuthu S: Medicinal plants used by traditional healers in Kancheepuram District of Tamil Nadu, India. J Ethnobiol Ethnomed 2006, 2:43.

77. Zenebe G, Zerihun M, Solomon Z: An ethnobotanical study of medicinal plants in Asgede Tsimbila District, Northwestern Tigray, Northern Ethiopia. Ethnobotany Research \& Applications 2012, 10:305-320

78. Addis $G$, Abebe $D$, Urga K: A survey of traditional medicinal plants in Shirka District, Arsi Zone, Ethiopia. Ethiop Pharm J 2001, 19:30-47.

79. Balemie K, Kelbessa E, Asfaw Z: Indigenous medicinal plant utilization, management and threats in Fentalle area, Eastern Shewa, Ethiopia. Ethiop J Biol Sci 2004, 3:37-58.

80. Tolossa K, Debela E, Athanasiadou S, Tolera A, Ganga G, Houdijk JGM: Ethno-medicinal study of plants used for treatment of human and livestock ailments by traditional healers in South Omo, Southern Ethiopia. J Ethnobiol Ethnomed 2013, 9:32.

81. Van Andel T, Mitchell S, Volpato G, Vandebroek I, Swier J, Ruysschaer S, Jiménez CAR, Raes N: In search of the perfect aphrodisiac: parallel use of bitter tonics in West Africa and the Caribbean. J Ethnopharmacol 2012, 143:840-850.

82. Voeks RA: Traditions in transition: african diaspora ethnobotany in lowland South America. In Mobility and Migration in Indigenous Amazonia: Contemporary Ethnoecological Perspectives. Edited by Alexiades M. London: Beghahn Books; 2009:275-294.

83. Sheldon JW, Balick MJ, Laird SA: Medicinal plants: can utilization and conservation coexist? Advances in Economic Botany 1997, 12:1-104.
84. Teklehaymanot $\mathrm{T}$ : Ethnobotanical study of knowledge and medicinal plants use by people in Dek Island in Ethiopia. J Ethnopharmacol 2009, 124:69-78.

85. Silva FDS, Ramos MA, Hanazaki N, Albuquerque UP: Dynamics of traditional knowledge of medicinal plants in a rural community in the Brazilian semi-arid region. Brazilian Journal of Pharmacognosy 2011, 21:382-391.

86. Giday M, Asfaw Z, Woldu Z, Teklehaymanot T: Medicinal plant knowledge of the Bench Ethnic group of Ethiopia: an ethnobotanical investigation. J Ethnobiol Ethnomed 2009, 5:34.

87. Begossi A, Hanazaki N, Tamashiro JY: Medicinal plants in the Atlantic Forest (Brazil): knowledge, use and conservation. Hum Ecol 2002, 30:281-299.

88. Uniyal SK, Singh KN, Jamwal P, Lal B: Traditional use of medicinal plants among the tribal communities Chhota Bhangal, Western Himalaya. J Ethnobiol Ethnomed 2006, 2:14

89. Voeks RA: Are women reservoirs of traditional plant knowledge? Gender, ethnobotany and globalization in northeast Brazil. Singap J Trop Geogr 2007, 28:7-20.

90. Giday M, Teklehaymanot T, Animut A, Mekonnen Y: Medicinal plants of the Shinasha, Agew-awi and Amhara peoples in northwest Ethiopia. J Ethnopharmacol 2007, 110:516-525.

91. Hussain A, Khan MN, Iqbal Z, Sajid MS: An account of the botanical anthelmintics used in traditional veterinary practices in Sahiwal district of Punjab, Pakistan. J Ethnopharmacol 2008, 2008(119):185-190

92. Abebe $D$ : The role of medicinal plants in healthcare coverage of Ethiopia, the possible benefits of integration. In Proceedings of the National workshop on Biodiversity Conservation and Sustainable Use of Medicinal Plants in Ethiopia: 28 April-1May 1998. Edited by Zewdu M, Demissie A. Addis Ababa, Ethiopia: Institute of Biodiversity Conservation and Research; 2001:6-21

93. Kloos $\mathrm{H}$, Tekle $\mathrm{A}$, Yohannes $\mathrm{L}$, Yosef $\mathrm{A}$ : Preliminary studies of traditional medicinal plants in nineteen markets in Ethiopia: use patterns and public health aspects. Ethiop Med J 1978, 16:33-43.

94. Dessissa D: A Preliminary economic valuation of medicinal plants in Ethiopia: trade, volume and price. In Proceedings of Workshop on Biodiversity Conservation and Sustainable Use of Medicinal Plants in Ethiopia. Edited by Zewdu M, Demissie A. Addis Ababa, Ethiopia: Institute of Biodiversity Conservation and Research; 2001:176-187.

95. Kloos H: Preliminary studies of medicinal plants and plant products in markets of central Ethiopia. Ethnomedicine 1976, 4:63-102.

96. Mander M, Emana B, Asfaw Z, Busa B: Marketing of Medicinal Plants in Ethiopia: A Survey of the Trade in Medicinal Plants: A research report prepared by Sustainable Use of medicinal Plants Project. Addis Ababa, Ethiopia: IBC; 2006

97. Trotter RT, Logan MH: Informants consensus: a new approach for identifying potentially effective medicinal plants. In Plants in Indigenous Medicine and Diet. Edited by Etkin NL. New York: Redgrave Publishing Company, Bedford Hill; 1986:91-112.

doi:10.1186/1746-4269-10-2

Cite this article as: Lulekal et al: Ethnoveterinary plants of Ankober District, North Shewa Zone, Amhara Region, Ethiopia. Journal of Ethnobiology and Ethnomedicine 2014 10:21.

\section{Submit your next manuscript to BioMed Central and take full advantage of:}

- Convenient online submission

- Thorough peer review

- No space constraints or color figure charges

- Immediate publication on acceptance

- Inclusion in PubMed, CAS, Scopus and Google Scholar

- Research which is freely available for redistribution 